з SUPPLEMENTARY MATERIAL

5 The influence of particle composition upon the evolution of

6 urban ultrafine diesel particles on the neighbourhood scale

9 Irina Nikolova, Xiaoming Cai, Mohammed Salim Alam,

10 Soheil Zeraati-Rezaei, Jian Zhong, A. Rob MacKenzie and

11 Roy M. Harrison 
14 Here we present details of the input parameters used in the box model simulations (Section S1)

15 and some results (Section S2). All figures and tables are given in the Appendix.

16

17 S1. Model input parameters - size distribution, vapour pressures, modal composition mass

18 fractions in the nucleation and Aitken modes, gas-phase ambient concentrations.

20 The initial size distribution is based on the measurements of Dall'Osto et al. (2011) and it is

21 plotted in Figure 1-S. This ultrafine size resolved distribution represents the typical street canyon

22 size distribution found next to a traffic site in Marylebone road in London (UK). The distribution

23 has a well defined nucleation mode (particles with a diameter around or less than $30 \mathrm{~nm}$ ) with a

24 peak number concentration at 23 - $24 \mathrm{~nm}$. The Aitken mode (particles with diameter between

2530 and $100 \mathrm{~nm}$ ) is seen as a shoulder attached to the nucleation mode with a centre between 50 -

$2660 \mathrm{~nm}$. Dall'Osto et al. (2011) show that the observed size distribution is subject to a major

27 transformation caused by extensive evaporation of volatile material from the particles. The

28 diameter of the nucleation mode particles decreased during the transport of the particles between

29 the street canyon and the nearby city park over a distance of about $665 \mathrm{~m}$, as shown in Figure 1-S

30 in the Appendix. The nucleation-mode peak diameter, $\mathrm{D}_{\mathrm{pg}, \mathrm{nuc}}$, corresponding to the highest number

31 concentration in the nucleation mode, was found at around $8-9 \mathrm{~nm}$. In our study, we aim to put

32 forward a realistic set of compositions and thermodynamic properties that could explain this

33 diameter decrease as seen in the observations. Chemical analyses during the observations are

34 missing; however, there are some laboratory data pointing to the nature of organics that

35 participate in the composition from emitted particles collected from an engine testbed (Alam et

36 al., 2016). Figure 2-S shows the normalised mass concentration per compound in the range

$37\left[\mathrm{C}_{16} \mathrm{H}_{34}: \mathrm{C}_{32} \mathrm{H}_{66}\right]$ for a given size class. Particles in the Nucleation and Aitken modes consist of

38 about $10 \%$ and higher contribution from compounds in the range $\mathrm{C}_{25} \mathrm{H}_{52}-\mathrm{C}_{29} \mathrm{H}_{60}$. 
40

41

42

43

44

45

46

$47 \quad i=1, \ldots, 15-$ number of size bins

$48 j=1, \ldots, 18$ - indices [1-17] correspond to surrogate n-alkane compounds in the range $\mathrm{C}_{16} \mathrm{H}_{34}-$

$49 \mathrm{C}_{32} \mathrm{H}_{66}$; index 18 refers to the involatile core

$50 k=1,2$ - particle mode, where 1 = Nucleation mode, 2 = Aitken mode

$51 D p_{i}$ - particle diameter per size bin $i, \mathrm{~m}$

$52 M_{i j}$ - size resolved mass per size bin i and per compound $j, \mathrm{~kg} \mathrm{~m}^{-3}$

$53 \rho=1000 \mathrm{~kg} \mathrm{~m}^{-3}$, particle density, held constant to avoid introducing highly uncertain parameters

54 for density of involatile core.

$55 X_{j k}$ - fraction of mass in a particle in mode $k$ per compound $j$

$56 \pi=3.14$

57 is the number of particles per bin width $\Delta D p,\left[\# \mathrm{~m}^{-3}\right]$. The number log-normal size distribution

58 (eq. 2) is given as follows:

59

60

$$
n(D p)_{i k}=\frac{N_{k}}{\sqrt{(2 \pi) D p_{i} \ln \left(\sigma_{g k}\right)}} \exp \left(-0.5\left(\frac{\ln \left(D p_{i} / D p_{g k}\right)}{\ln \left(\sigma_{g k}\right)}\right)^{2}\right)
$$

$63 N_{k}=3.0 \times 10^{10}$ and $1.8 \times 10^{10}\left(\# \mathrm{~m}^{-3}\right)$ are the total number concentrations in the Nucleation and

64 Aitken mode, respectively 
$D p_{g k}=23$ and $65 \mathrm{~nm}$ are the geometric mean diameter in the Nucleation and Aitken mode,

66 respectively

$67 \quad \sigma_{\mathrm{gk}}=1.6$ is the geometric standard deviation for both modes

68 The fraction of mass $X_{j k}$ of each particle in a mode $k$ per compound SVOC in the range $\mathrm{C}_{16} \mathrm{H}_{34}{ }^{-}$

$69 \mathrm{C}_{32} \mathrm{H}_{66}$ is calculated (eq. 3) as follows:

70

$$
X_{j k}=\frac{f\left(m_{j}, \sigma_{\text {nodal }}\right)}{\sum_{j=1}^{17} f\left(m_{j}, \sigma_{n o d a l}\right)}\left(x x_{j k}-s f_{k}\right)
$$

72

73 where $f\left(m_{j}, \sigma_{\text {modal }}\right)$ is the modal composition in the form of a Gaussian distribution (eq. 4$), x x_{j k}$ is

74 SVOC composition given by the Gaussian parameterisation (eq. 5), and $s f_{k}$ is the solid involatile

75 fraction per mode $k$ :

76

$$
f\left(m_{j}, \sigma_{\text {modal }}\right)=\frac{1}{\sqrt{2 \pi} \sigma_{\text {modal }}} \exp \left(-0.5\left(\frac{\left(x_{j}-m_{j}\right)}{\sigma_{\text {modal }}}\right)^{2}\right)
$$

78

$$
x x_{j k}=\sum_{j=1}^{17} \frac{f\left(m_{j}, \sigma_{\text {modal }}\right)}{\sum_{j=1}^{17} f\left(m_{j}, \sigma_{\text {modal }}\right)}=1
$$

$\sigma_{\text {modal }}=1, \ldots, 5-$ standard deviation of modal composition

$x_{j}=1, \ldots, 17-$ number assigned to the SVOC compound

83

$m_{j}=1, \ldots, 17$ - modal composition compound for each SVOC in the range $\mathrm{C}_{16} \mathrm{H}_{34}-\mathrm{C}_{32} \mathrm{H}_{66}$

84

85 In this study the role of an involatile core $\left(s f_{k}\right)$ is evaluated, too, by considering an involatile core

86 to be $1 \%, 5 \%$ and $10 \%$ of the mass in a particle in the Nucleation mode $(k=1)$. Input modal 
composition mass fractions in the nucleation mode and composition standard deviation are presented in the Appendix in Table 1-S, 2-S and 3-S for involatile core of 1\%, 5\% and 10\%, respectively. The involatile core in the Aitken mode is $90 \%$ and the input modal composition mass fractions are given in Table 4-S in the Appendix.

Input vapour pressure parameterisations are given in Table 5-S. A-a, B-c and Co are used in this study to represent the uncertainties in the vapour pressure and evaluate the overall effect on the evaporative shrinkage of the nucleation mode particle diameter. Figure 3-S shows the ratio of vapour pressure between $298 \mathrm{~K}$ and $273 \mathrm{~K}$ for A-a, B-c and Co vapour pressure parameterisations and n-alkane compounds in the range $\mathrm{C}_{16} \mathrm{H}_{34}-\mathrm{C}_{32} \mathrm{H}_{66}$. The ratio for $\mathrm{B}-\mathrm{c}$ and Co vapour pressure parameterisations is within an order of magnitude for compounds in the range $\mathrm{C}_{16} \mathrm{H}_{34}-\mathrm{C}_{25} \mathrm{H}_{52}$, however, it increases two to three orders of magnitude for higher molecular weight compounds.

The ratio for A-a vapour pressure parameterisation is within an order of magnitude for compounds in the range $\mathrm{C}_{16} \mathrm{H}_{34}-\mathrm{C}_{21} \mathrm{H}_{44}$, and increases substantially for the remaining compounds. This would imply that for the selected timescale of $100 \mathrm{~s}$ there will be a shift in the threshold modal compositions to lower carbon-number compounds in comparison with the threshold modal compositions discussed in this study. The temperature dependence on evaporation is not considered further in this study but should be borne in mind.

The initial gas-phase concentrations for the n-alkanes in the range $\mathrm{C}_{16} \mathrm{H}_{34}-\mathrm{C}_{32} \mathrm{H}_{66}$ (Table 6-S) are as for the study in Nikolova et al. (2016) and are based on the roadside atmospheric measurements of Harrad et al. (2003).

\section{S2. Results}

Figure 4-S and Figure 5-S show the nucleation mode peak diameter $D_{\mathrm{pg}, \text { nuc }}$ after $100 \mathrm{~s}$ using the vapour pressure parameterisations following Myrdal-Yalkowsky et al (1997, B-c) and Nannoolal 
et al (2008, A-a), respectively. The nucleation mode particles consist of initial $1 \%$ non-volatile material. The threshold modal composition value changes from $\mathrm{C}_{27} \mathrm{H}_{56}$ for the $\mathrm{B}-\mathrm{c}$ parameterisation (Figure 4-S) to $\mathrm{C}_{22} \mathrm{H}_{46}$ for A-a (Figure 5-S).

Figure 6-S shows the relative difference of the $\mathrm{D}_{\mathrm{pg}, \mathrm{nuc}}$ between the highest $(\mathrm{B}-\mathrm{c})$ and lowest $(\mathrm{A}-\mathrm{a})$ vapour pressure parameterisations evaluated at $1 \mathrm{~s}, 10 \mathrm{~s}, 50 \mathrm{~s}$ and $100 \mathrm{~s}$. Overall the largest difference is propagating to higher standard deviation sigma when simulation time increases as well as moving towards higher carbon-number modal compositions. In other words the relative differences become larger with time, pointing back to the huge differences in vapour pressure parameterisations between B-C and A-a. The choice of a particular vapour pressure dataset changes the range of carbon numbers by 2 in the first 10 seconds for which the highest relative difference is simulated. The 1-s relative difference is the highest for sigma 1 and modal compositions $\mathrm{C}_{19} \mathrm{H}_{40}-\mathrm{C}_{21} \mathrm{H}_{44}$. The 10-s highest relative difference has shifted to modal compositions $\mathrm{C}_{21} \mathrm{H}_{44}-\mathrm{C}_{23} \mathrm{H}_{48}$ and sigma $=1$, 2. Higher relative differences (50\% and more) are also simulated at sigma $=3$. The 100 -s relative difference is the highest for modal compositions $\mathrm{C}_{22} \mathrm{H}_{46}-\mathrm{C}_{24} \mathrm{H}_{50}$ and sigma $=1,2$, 3, but also relative differences of around $50 \%$ are simulated for sigma $=5$.

Figure 7-S shows the '100-s effective involatile core' for the nucleation mode particles. Results are shown at $1 \%, 5 \%$ and $10 \%$ initial non-volatile material in the nucleation mode particles, modal compositions $\mathrm{C}_{24} \mathrm{H}_{34}$ and $\mathrm{C}_{32} \mathrm{H}_{66}$ and for various composition standard deviations, sigma. Vapour pressure parameterisations follow Myrdal and Yalkowski (1997; B-c in Table 1-S) and Nannoolal et al. (2008; A-a in Table 1-S). Figure 5-S presents the sensitivity to the non-volatile core in the nucleation mode for modal compositions $\mathrm{C}_{24} \mathrm{H}_{50}$ and $\mathrm{C}_{32} \mathrm{H}_{66}$ evaluated for B-c and A-a vapour pressure parameterisations. The 100-s effective involatile core for modal composition $\mathrm{C}_{24} \mathrm{H}_{50}$ and B-c vapour pressure parameterisation increases when the non-volatile core increases. 
This is simulated for an increasing $\sigma$ too, due the increasing number of lower volatility components that are added into the particle composition. The 100-s effective involatile core for modal compositions $\mathrm{C}_{24} \mathrm{H}_{50}$ (for A-a vapour pressure) and $\mathrm{C}_{32} \mathrm{H}_{66}$ (for A-a and B-c vapour pressures) shows an opposite trend with respect to sigma, i.e., the 100-s effective involatile core decreases due to the increasing number of higher volatility components added into the particle composition.

\title{
Acknowledgements
}

This work is part of the FASTER project, ERC-2012-AdG, Proposal No. 320821 sponsored by the European Research Council (ERC).

\section{References}

\author{
Alam, M. S, Rezaei, S. Z., Stark, C. P., Liang, Z., Xu, H. M., Harrison, R. M.: The
} characterisation of diesel exhaust particles - composition, size distribution and partitioning, Faraday Discuss., 189, 69-84, 2016.

Chickos, J., Lipkind, D.: Hypothetical thermodynamic properties: vapour pressures and vaporization enthalpies of the even n-Alkanes from C78 to C92 at T=298.15K by correlation-gas chromatography, J. Chem. Eng. Data, 53, 2432-2440, 2008.

Compernolle, S., Ceulemans, K., Muller, J. -F.: EVAPORATION: a new vapour pressure estimation method for organic molecules including non-additivity and intramolecular interactions, Atmos. Chem. Phys., 11, 9431-9450, 2011.

\footnotetext{
Dall’Osto, M., Thorpe, A., Beddows, D.C.S., Harrison, R.M., Barlow, J.F., Dunbar, T., Williams,
} 
P.I., Coe, H.: Remarkable dynamics of nanoparticles in the urban atmosphere, Atmos. Chem. Phys., 11, 6623-6637, 2011.

Joback, K., Reid, R.: Estimation of pure-component properties from group-contributions, Chem. Eng. Commun., 57, 233-243, 1987.

Harrad, S., Hassoun, S., Callen Romero, M. S., Harrison, R. M.: Characterisation and source attribution of the semi-volatile organic content of atmospheric particles and associate vapour phase in Birmingham, UK , Atmos. Environ., 37, 4985-4991, 2003.

Myrdal, P. B., Yalkowsky, S. H.: Estimating pure component vapor pressures of complex organic molecules, Ind. Eng. Chem. Res., 36, 2494-2499, 1997.

Nannoolal, Y., Rarey, J., Ramjugernath, D., Cordes, W.: Estimation of pure component properties: Part 1. Estimation of the normal boiling point of non-electrolyte organic compounds via group contributions and group interactions, Fluid Phase Equilibr., 226, 45-63, 2004.

Nannoolal, Y., Rarey, J., Ramjugernath, D.: Estimation of pure component properties: Part 3. Estimation of the vapor pressure of non-electrolyte organic compounds via group contributions and group interactions, Fluid Phase Equilibr., 269, 117-133, 2008.

Nikolova, I., MacKenzie, A. R., Cai, X., Alam, M. S., Harrison, R. M.: Modelling component evaporation and coposition change of traffic-induced ultrafine particles during travel from street canyon to urban background, Faraday Discuss., 189, 529-46, 2016.

Stein, S. E., Brown, R. L.: Estimation of normal boiling points from group contributions, J. 
191 Chem. Inf. Comp. Sci., 34, 581-587, 1994.

192

193 Topping, D., Barley, M., Bane, M. K., Higham, N., Aumont, B., Dingle, N., McFiggans, G.:.

194 UManSysProp v1.0: an online and open-source facility for molecular property prediction and

195 atmospheric aerosol calculations, Geosci. Model Dev.., 9, 899-914, 2016.

196

197

198

199

200

201

202

203

204

205

206

207

208

209

210

211

212

213

214

215

216 


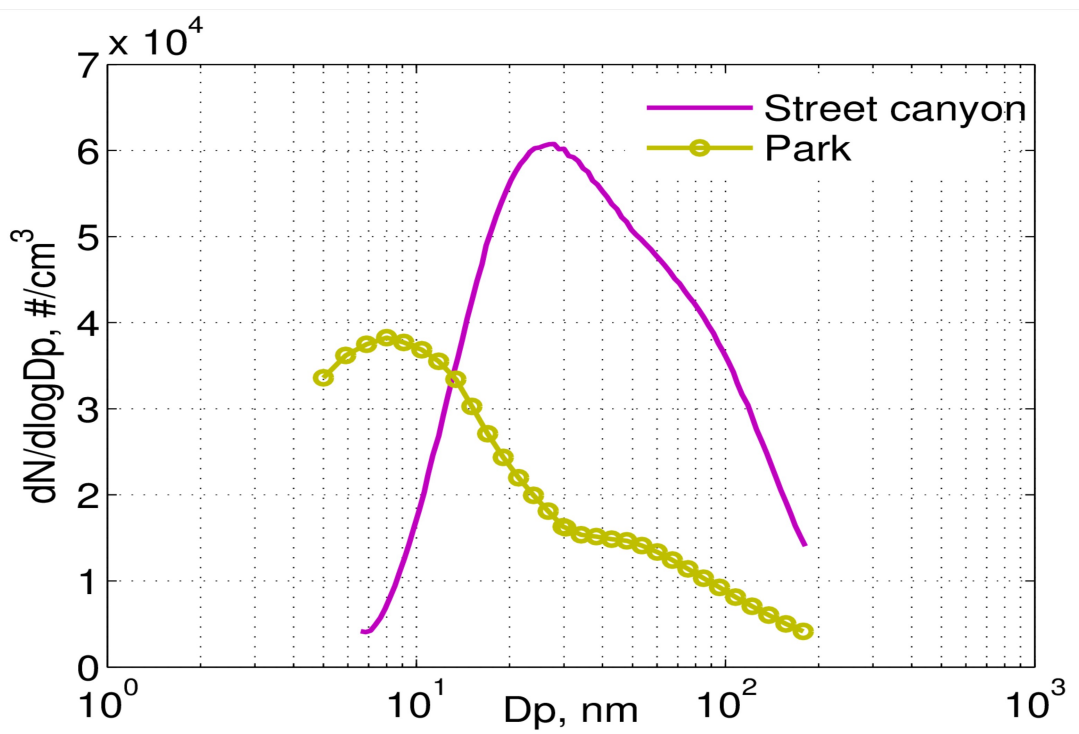

229

230

Figure 1-S. Measured ultrafine particle size distribution in a street canyon (Marylebone Road, 


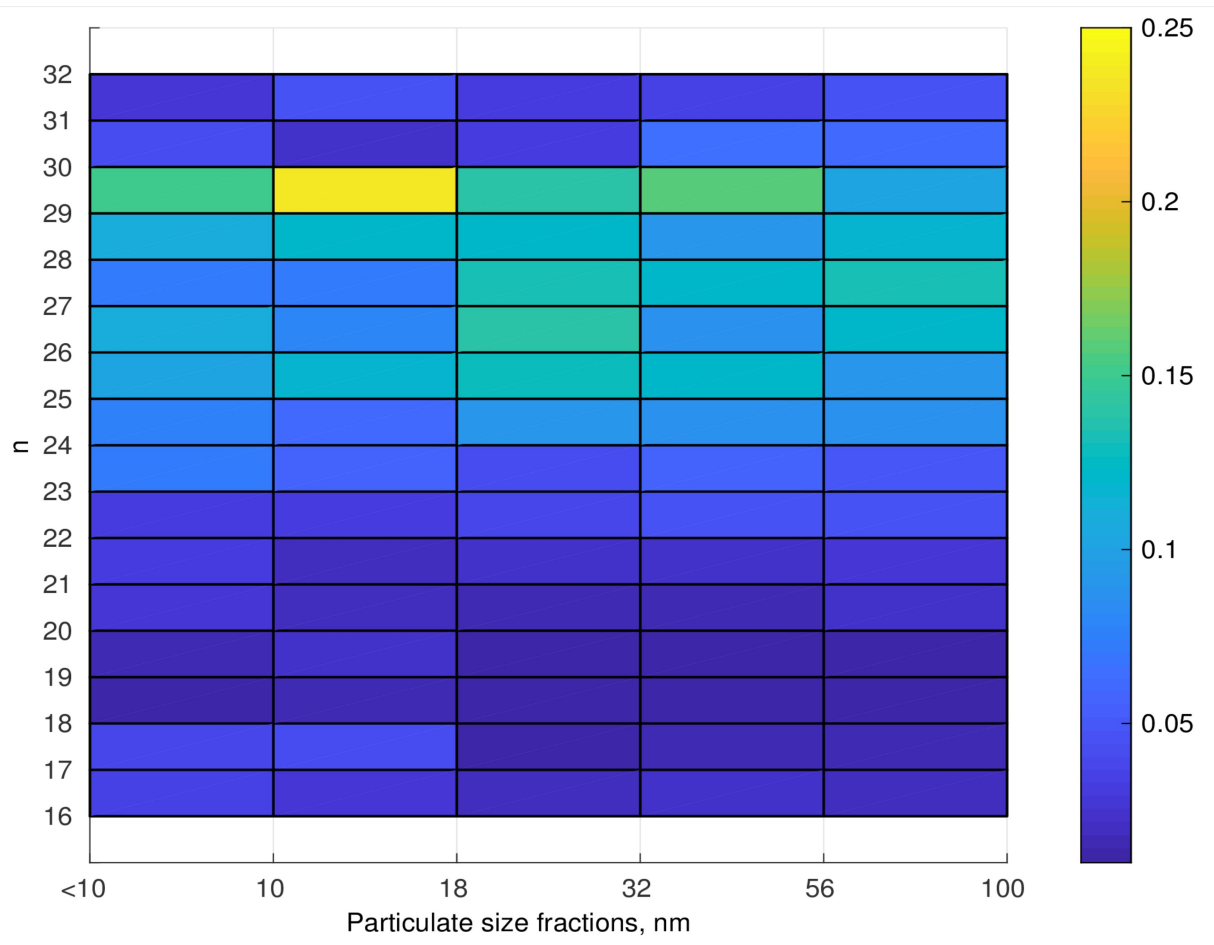

255

256

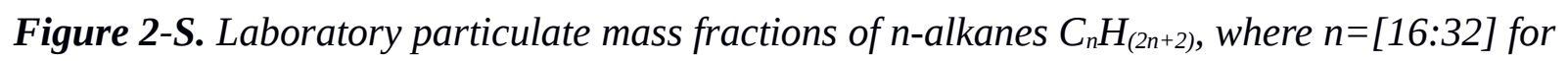
258 selected particulate diameter range [ $<10: 100 \mathrm{~nm}]$. Figure plotted based on the data presented in Alam et al. (2016). 


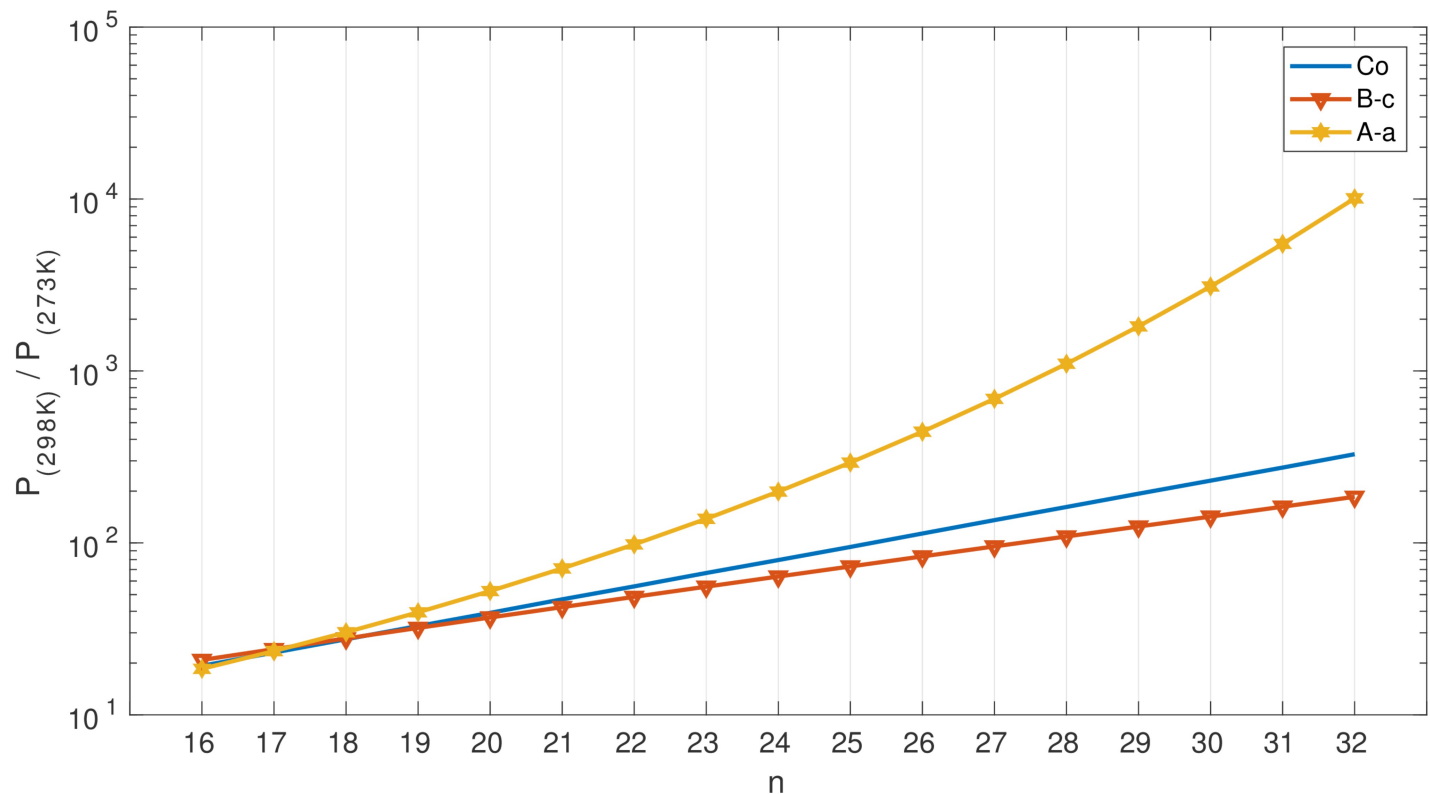

279

280

281

Figure 3-S. Vapour pressure ratios for vapour pressure parameterisations $A-a, B-c$ and Co and

283

284 


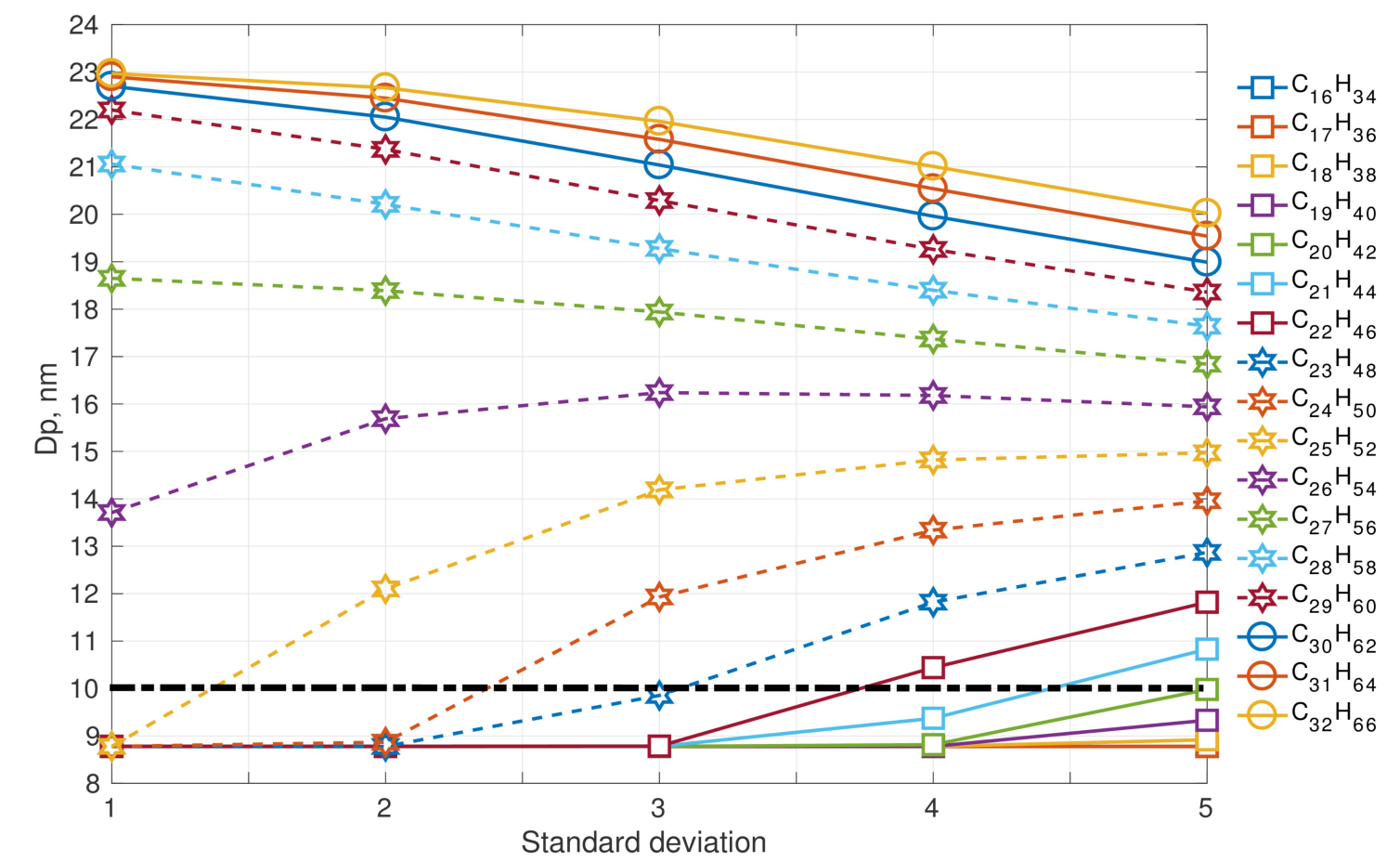

306

307

Figure 4-S. Nucleation mode peak diameter Dp [nm] at $100 \mathrm{~s}$ of simulation depending on the modal composition and the composition standard deviation. The initial nucleation mode peak diameter is at $23 \mathrm{~nm}$ (not shown on the figure). Vapour pressure data follows Myrdal Yalkowsky et al. $(1997, B-c)$. Initial nucleation mode particle involatile core is $1 \%$. 


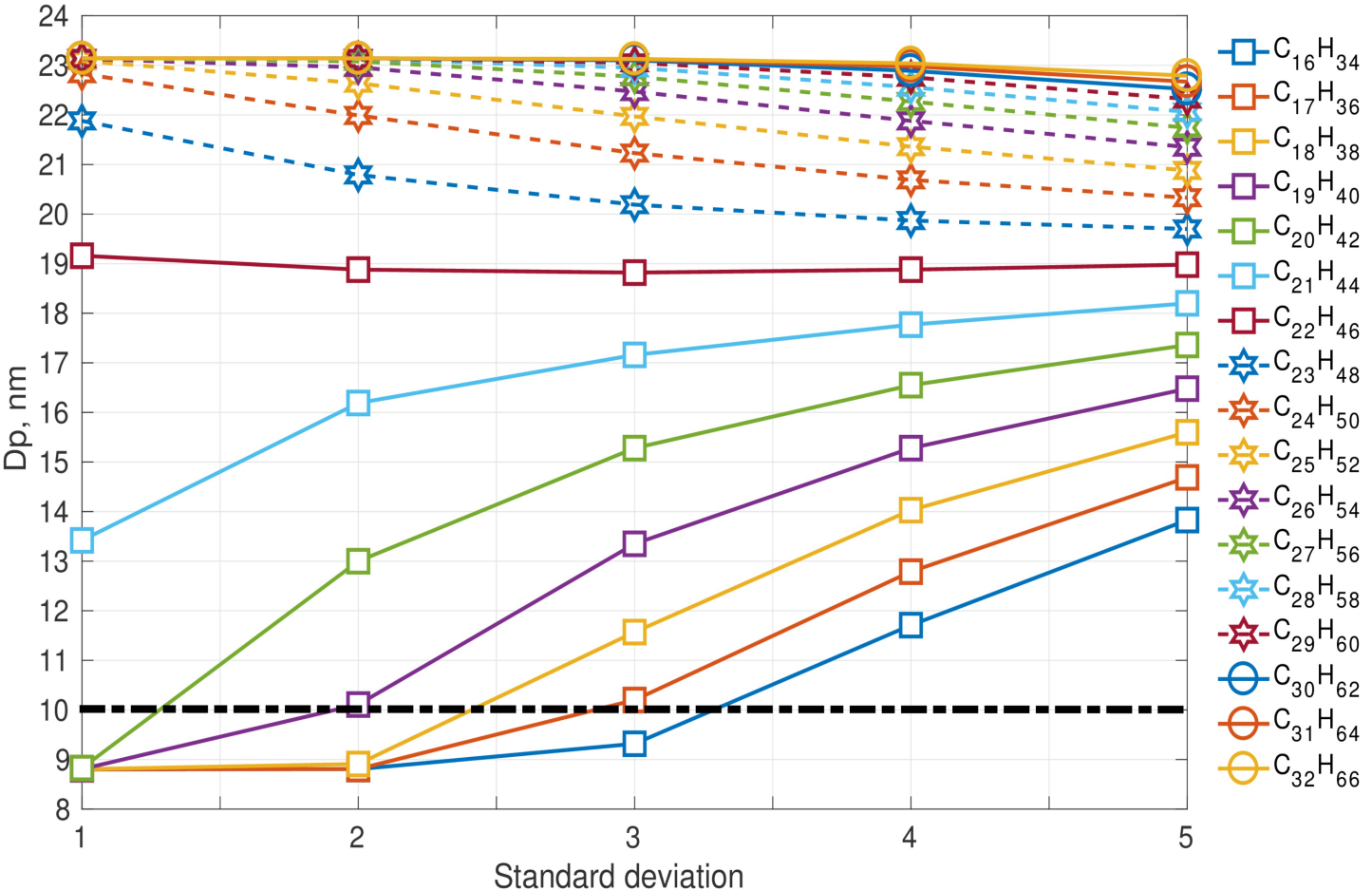

Standard deviation

Figure 5-S. Nucleation mode peak diameter Dp [nm] at $100 \mathrm{~s}$ of simulation depending on the modal composition and the composition standard deviation. The initial nucleation mode peak diameter is at $23 \mathrm{~nm}$ (not shown on the figure). Vapour pressure data follows Nannoolal et al. (2008, A-a). Initial nucleation mode particle involatile core is $1 \%$. 

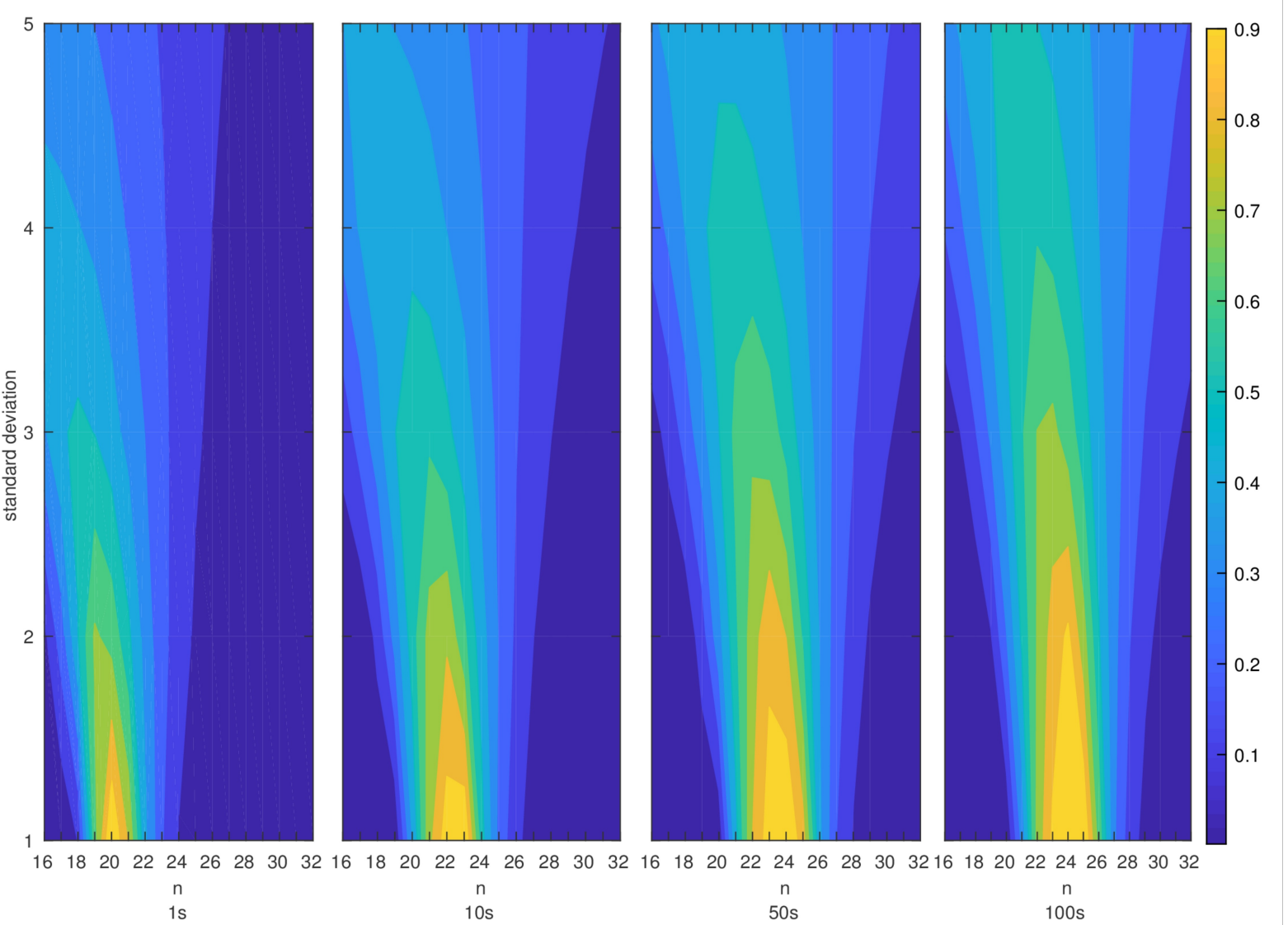

1

3

54

55

Figure 6-S. Nucleation mode peak diameter relative difference (in \%) between B-c and A-a 

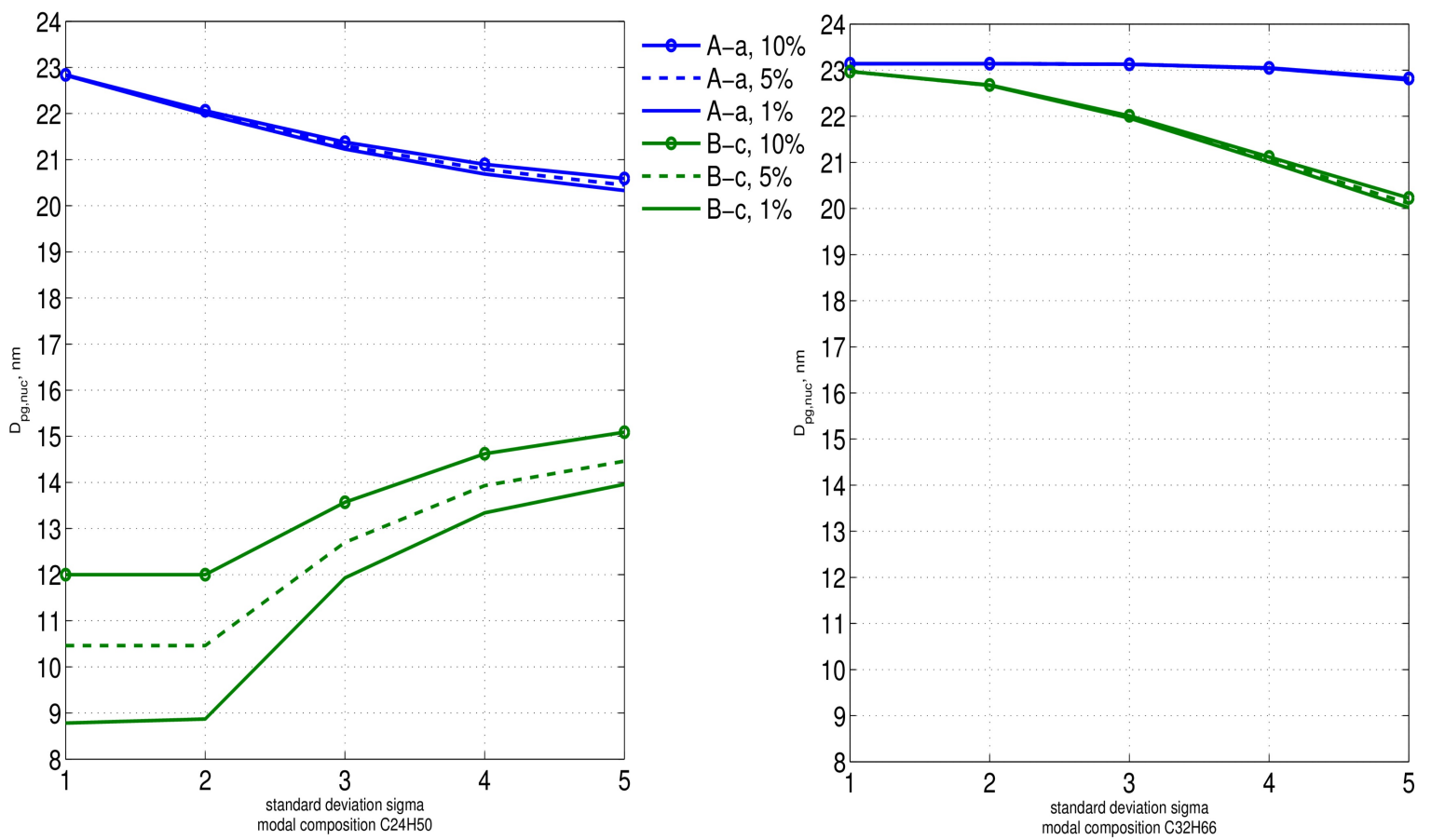

Figure 7-S. Nucleation mode peak diameter Dp [nm] after 100s: the '100-s effective involatile core' for the nucleation mode. Results are shown at 1\%, 5\% and 10\% initial non-volatile material in the nucleation mode particles, modal compositions $\mathrm{C}_{24} \mathrm{H}_{34}$ (left) and $\mathrm{C}_{32} \mathrm{H}_{66}$ (right) and for various composition standard deviations, sigma. Vapour pressure parameterisation follows Myrdal and Yalkowski (1997; B-c in Table 1-S) and Nannoolal et al. (2008; A-a in Table 1-S). 


\begin{tabular}{|c|c|c|c|c|c|c|c|c|c|c|c|c|c|c|c|c|}
\hline \multicolumn{17}{|c|}{ modal composition } \\
\hline $5.65 \mathrm{E}-01$ & $2.54 \mathrm{E}-01$ & $5.37 \mathrm{E}-02$ & 4.39E-03 & $1.32 \mathrm{E}-04$ & $1.47 \mathrm{E}-06$ & $6.02 \mathrm{E}-09$ & $9.04 \mathrm{E}-12$ & $5.00 \mathrm{E}-15$ & $1.02 \mathrm{E}-18$ & $7.62 \mathrm{E}-23$ & $2.10 \mathrm{E}-27$ & $2.12 \mathrm{E}-32$ & $7.92 \mathrm{E}-38$ & $1.09 \mathrm{E}-43$ & $5.82 \mathrm{E}-50$ & $1.45 E-56$ \\
\hline 3.42E-01 & 4.20E-01 & $2.41 \mathrm{E}-01$ & $5.35 \mathrm{E}-02$ & 4.39E-03 & 1.32E-04 & $1.47 \mathrm{E}-06$ & $6.02 \mathrm{E}-09$ & $9.04 \mathrm{E}-12$ & $5.00 \mathrm{E}-15$ & $1.02 \mathrm{E}-18$ & $7.62 \mathrm{E}-23$ & $2.10 \mathrm{E}-27$ & 2.13E-32 & $7.96 \mathrm{E}-38$ & $1.15 \mathrm{E}-43$ & $7.83 \mathrm{E}-50$ \\
\hline $7.64 \mathrm{E}-02$ & $2.54 \mathrm{E}-01$ & $3.97 \mathrm{E}-01$ & 2.40E-01 & $5.35 \mathrm{E}-02$ & 4.39E-03 & $1.32 \mathrm{E}-04$ & $1.47 \mathrm{E}-06$ & $6.02 \mathrm{E}-09$ & $9.04 \mathrm{E}-12$ & $5.00 \mathrm{E}-15$ & $1.02 \mathrm{E}-18$ & $7.62 \mathrm{E}-23$ & 2.10E-27 & $2.13 E-32$ & $8.41 \mathrm{E}-38$ & $1.55 \mathrm{E}-43$ \\
\hline 2.10E-06 & $1.41 \mathrm{E}-04$ & 4.41E-03 & $5.35 \mathrm{E}-02$ & 2.40E-01 & 3.95E-01 & $2.40 \mathrm{E}-01$ & $5.35 \mathrm{E}-02$ & $4.39 \mathrm{E}-03$ & $1.32 \mathrm{E}-04$ & 1.47E-06 & $6.02 \mathrm{E}-09$ & $9.04 \mathrm{E}-12$ & $5.00 \mathrm{E}-15$ & $1.02 \mathrm{E}-18$ & $8.09 \mathrm{E}-23$ & $3.00 \mathrm{E}-27$ \\
\hline $8.60 \mathrm{E}-09$ & $1.56 \mathrm{E}-06$ & $1.33 \mathrm{E}-04$ & 4.39E-03 & $5.35 \mathrm{E}-02$ & 2.40E-01 & $3.95 \mathrm{E}-01$ & 2. $40 \mathrm{E}-01$ & $5.35 \mathrm{E}-02$ & $4.39 \mathrm{E}-03$ & $1.32 \mathrm{E}-04$ & $1.47 \mathrm{E}-06$ & $6.02 \mathrm{E}-09$ & $9.04 \mathrm{E}-12$ & $5.02 \mathrm{E}-15$ & $1.08 \mathrm{E}-18$ & $1.09 \mathrm{E}-22$ \\
\hline 1.29E-11 & $6.39 \mathrm{E}-09$ & $1.48 \mathrm{E}-06$ & $1.33 \mathrm{E}-04$ & 4.39E-03 & $5.35 \mathrm{E}-02$ & $2.40 \mathrm{E}-01$ & 3.95E-01 & $2.40 \mathrm{E}-01$ & $5.35 \mathrm{E}-02$ & $4.39 \mathrm{E}-03$ & $1.32 \mathrm{E}-04$ & $1.47 \mathrm{E}-06$ & $6.02 \mathrm{E}-09$ & $9.08 \mathrm{E}-12$ & $5.31 \mathrm{E}-15$ & $1.45 \mathrm{E}-18$ \\
\hline $3.00 \mathrm{E}-27$ & $8.09 E-23$ & $1.02 \mathrm{E}-18$ & $5.00 \mathrm{E}-15$ & $9.04 \mathrm{E}-12$ & $6.02 \mathrm{E}-09$ & $1.47 \mathrm{E}-06$ & $1.32 \mathrm{E}-04$ & $4.39 \mathrm{E}-03$ & $5.35 \mathrm{E}-02$ & $2.40 \mathrm{E}-01$ & $3.95 \mathrm{E}-01$ & $2.40 \mathrm{E}-01$ & $5.35 \mathrm{E}-02$ & $4.41 \mathrm{E}-03$ & $1.41 \mathrm{E}-04$ & 2.10E- 06 \\
\hline 3.04E-32 & $2.23 \mathrm{E}-27$ & 7.65E-23 & $1.02 \mathrm{E}-18$ & $5.00 \mathrm{E}-15$ & $9.04 \mathrm{E}-12$ & $6.02 \mathrm{E}-09$ & $1.47 \mathrm{E}-06$ & $1.32 \mathrm{E}-04$ & 4.39E-03 & $5.35 \mathrm{E}-02$ & 2. $40 \mathrm{E}-01$ & 3.95E-01 & 2. $40 \mathrm{E}-01$ & $5.37 \mathrm{E}-02$ & 4.66E-03 & $1.89 \mathrm{E}-04$ \\
\hline $1.13 E-37$ & $2.26 \mathrm{E}-32$ & $2.11 \mathrm{E}-27$ & $7.62 \mathrm{E}-23$ & $1.02 \mathrm{E}-18$ & $5.00 \mathrm{E}-15$ & $9.04 \mathrm{E}-12$ & $6.02 \mathrm{E}-09$ & $1.47 \mathrm{E}-06$ & $1.32 \mathrm{E}-04$ & $4.39 \mathrm{E}-03$ & $5.35 \mathrm{E}-02$ & $2.40 \mathrm{E}-01$ & 3.95E-01 & $2.41 \mathrm{E}-01$ & $5.68 \mathrm{E}-02$ & $6.27 \mathrm{E}-03$ \\
\hline $1.55 \mathrm{E}-43$ & $8.41 \mathrm{E}-38$ & $2.13 \mathrm{E}-32$ & $2.10 \mathrm{E}-27$ & $7.62 \mathrm{E}-23$ & $1.02 \mathrm{E}-18$ & $5.00 \mathrm{E}-15$ & $9.04 \mathrm{E}-12$ & $6.02 \mathrm{E}-09$ & 1.47E-06 & $1.32 \mathrm{E}-04$ & 4.39E-03 & $5.35 \mathrm{E}-02$ & 2. $40 \mathrm{E}-01$ & 3.97E-01 & $2.54 \mathrm{E}-01$ & 7.64E-02 \\
\hline 7.83E-50 & $1.15 \mathrm{E}-43$ & 7.96E-38 & 2.13E-32 & 2.10E-27 & 7.62E-23 & $1.02 \mathrm{E}-18$ & $5.00 \mathrm{E}-15$ & $9.04 \mathrm{E}-12$ & $6.02 \mathrm{E}-09$ & 1.47E-06 & $1.32 \mathrm{E}-04$ & 4.39E-03 & $5.35 \mathrm{E}-02$ & 2.41E-01 & 4.20E-01 & 3.42E-01 \\
\hline 1.45E-56 & $5.82 \mathrm{E}-50$ & 1.09E-43 & 7.92E-38 & 2.12E-32 & 2.10E-27 & 7.62E-23 & 1.02E-18 & $5.00 \mathrm{E}-15$ & $9.04 \mathrm{E}-12$ & $6.02 \mathrm{E}-09$ & 1.47E-06 & $1.32 \mathrm{E}-04$ & 4.39E-03 & $5.37 \mathrm{E}-02$ & 2.54E-01 & $5.65 \mathrm{E}-01$ \\
\hline & & & & & & & & & & & & & & & & \\
\hline
\end{tabular}

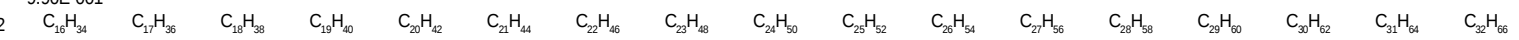

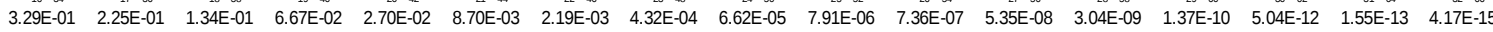

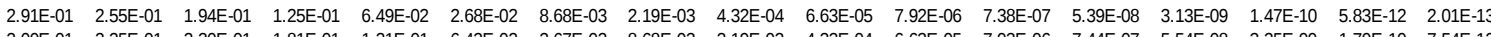

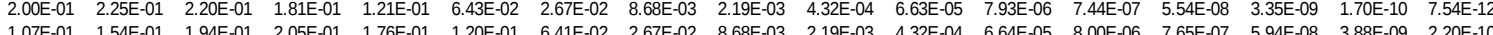

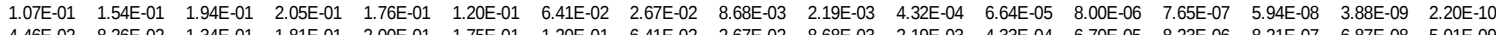

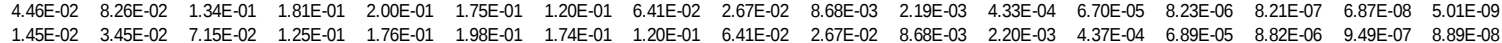
$\begin{array}{lllllllllllllllll}1.45 \mathrm{E}-02 & 3.45 \mathrm{E}-02 & 7.15 \mathrm{E}-02 & 1.25 \mathrm{E}-01 & 1.76 \mathrm{E}-01 & 1.98 \mathrm{E}-01 & 1.74 \mathrm{E}-01 & 1.20 \mathrm{E}-01 & 6.41 \mathrm{E}-02 & 2.67 \mathrm{E}-02 & 8.68 \mathrm{E}-03 & 2.20 \mathrm{E}-03 & 4.37 \mathrm{E}-04 & 6.89 \mathrm{E}-05 & 8.82 \mathrm{E}-06 & 9.49 \mathrm{E}-07 & 8.89 \mathrm{E}-08 \\ 3.66 \mathrm{E}-03 & 1.12 \mathrm{E}-02 & 2.98 \mathrm{E}-02 & 6.67 \mathrm{E}-02 & 1.21 \mathrm{E}-01 & 1.75 \mathrm{E}-01 & 1.98 \mathrm{E}-01 & 1.74 \mathrm{E}-01 & 1.20 \mathrm{E}-01 & 6.41 \mathrm{E}-02 & 2.67 \mathrm{E}-02 & 8.70 \mathrm{E}-03 & 2.22 \mathrm{E}-03 & 4.49 \mathrm{E}-04 & 7.39 \mathrm{E}-05 & 1.02 \mathrm{E}-05 & 1.23 \mathrm{E}-06 \\ 7.26 & & \end{array}$ $\begin{array}{lllllllllllllllll}3.66 \mathrm{E}-03 & 1.12 \mathrm{E}-02 & 2.98 \mathrm{E}-02 & 6.67 \mathrm{E}-02 & 1.21 \mathrm{E}-01 & 1.75 \mathrm{E}-01 & 1.98 \mathrm{E}-01 & 1.74 \mathrm{E}-01 & 1.20 \mathrm{E}-01 & 6.41 \mathrm{E}-02 & 2.67 \mathrm{E}-02 & 8.70 \mathrm{E}-03 & 2.22 \mathrm{E}-03 & 4.49 \mathrm{E}-04 & 7.39 \mathrm{E}-05 & 1.02 \mathrm{E}-05 & 1.23 \mathrm{E}-06 \\ 7.20 \mathrm{E}-04 & 2.83 \mathrm{E}-03 & 9.68 \mathrm{E}-03 & 2.78 \mathrm{E}-02 & 6.49 \mathrm{E}-02 & 1.20 \mathrm{E}-01 & 1.74 \mathrm{E}-01 & 1.97 \mathrm{E}-01 & 1.74 \mathrm{E}-01 & 1.20 \mathrm{E}-01 & 6.41 \mathrm{E}-02 & 268 \mathrm{E}-02 & 8.78 \mathrm{E}-03 & 2.28 \mathrm{E}-03 & 4.82 \mathrm{E}-04 & 8.54 \mathrm{E}-05 & 1.32 \mathrm{E}-05 \\ 1 & & \end{array}$

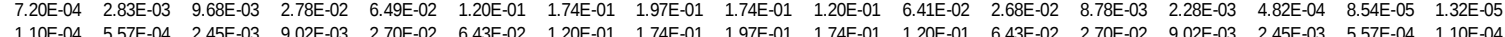

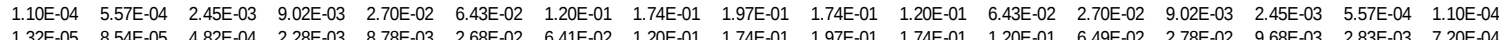
$\begin{array}{lllllllllllllllll}1.32 \mathrm{E}-05 & 8.54 \mathrm{E}-05 & 4.82 \mathrm{E}-04 & 2.28 \mathrm{E}-03 & 8.78 \mathrm{E}-03 & 2.68 \mathrm{E}-02 & 6.41 \mathrm{E}-02 & 1.20 \mathrm{E}-01 & 1.74 \mathrm{E}-01 & 1.97 \mathrm{E}-01 & 1.74 \mathrm{E}-01 & 1.20 \mathrm{E}-01 & 6.49 \mathrm{E}-02 & 2.78 \mathrm{E}-02 & 9.68 \mathrm{E}-03 & 2.83 \mathrm{E}-03 & 7.20 \mathrm{E}-04 \\ 1.23 \mathrm{E}-06 & 1.02 \mathrm{E}-05 & 7.39 \mathrm{E}-05 & 4.49 \mathrm{E}-04 & 2.22 \mathrm{E}-03 & 8.70 \mathrm{E}-03 & 2.67 \mathrm{E}-02 & 6.41 \mathrm{E}-02 & 1.20 \mathrm{E}-01 & 1.74 \mathrm{E}-01 & 1.98 \mathrm{E}-01 & 1.75 \mathrm{E}-01 & 1.21 \mathrm{E}-01 & 6.67 \mathrm{E}-02 & 2.98 \mathrm{E}-02 & 1.12 \mathrm{E}-02 & 3.66 \mathrm{E}-03 \\ 8.8 E & & \end{array}$

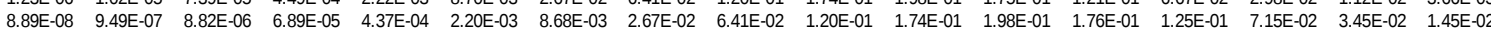

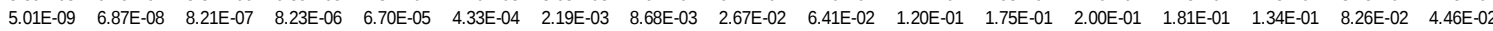
$\begin{array}{llllllllllllllllll}2.20 \mathrm{E}-10 & 3.88 \mathrm{E}-09 & 5.94 \mathrm{E}-08 & 7.65 \mathrm{E}-07 & 8.00 \mathrm{E}-06 & 6.64 \mathrm{E}-05 & 4.32 \mathrm{E}-04 & 2.19 \mathrm{E}-03 & 8.68 \mathrm{E}-03 & 2.67 \mathrm{E}-02 & 6.41 \mathrm{E}-02 & 1.20 \mathrm{E}-01 & 1.76 \mathrm{E}-01 & 2.05 \mathrm{E}-01 & 1.94 \mathrm{E}-01 & 1.54 \mathrm{E}-01 & 1.07 \mathrm{E}-01 \\ 7 & & & \end{array}$

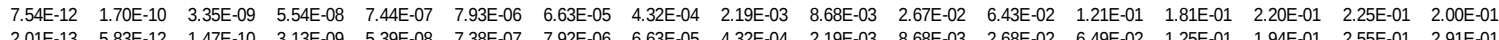

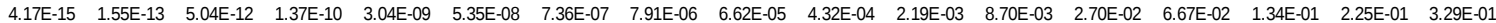

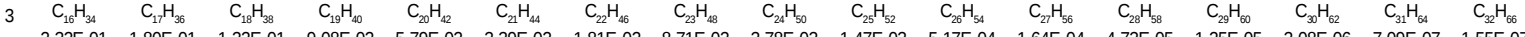

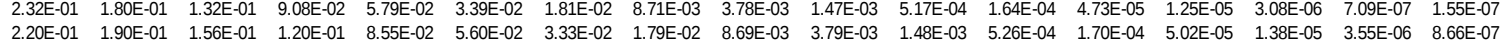

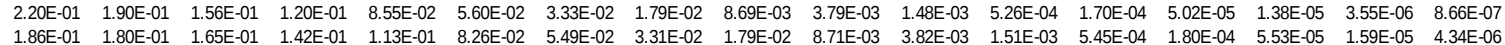

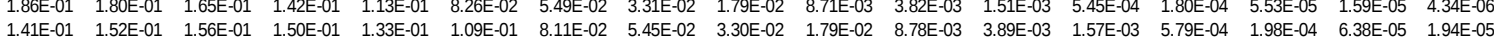

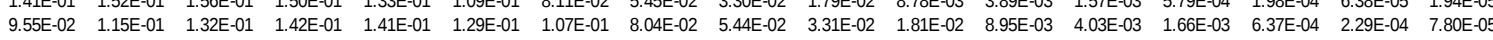

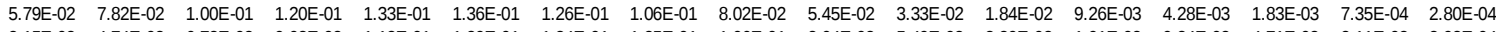

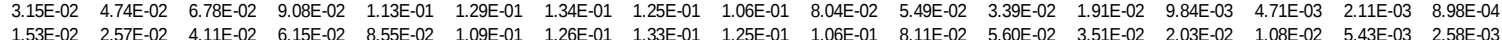

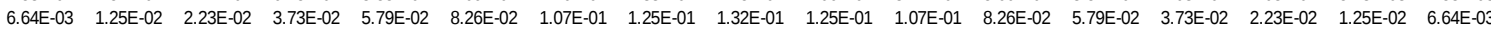

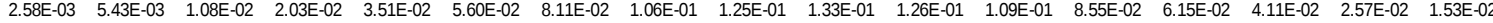

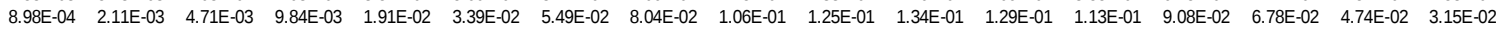

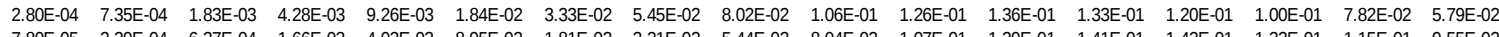

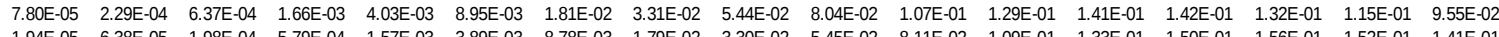
$\begin{array}{lllllllllllllllll}1.94 \mathrm{E}-05 & 6.38 \mathrm{E}-05 & 1.98 \mathrm{E}-04 & 5.79 \mathrm{E}-04 & 1.57 \mathrm{E}-03 & 3.89 \mathrm{E}-03 & 8.78 \mathrm{E}-03 & 1.79 \mathrm{E}-02 & 3.30 \mathrm{E}-02 & 5.45 \mathrm{E}-02 & 8.11 \mathrm{E}-02 & 1.09 \mathrm{E}-01 & 1.33 \mathrm{E}-01 & 1.50 \mathrm{E}-01 & 1.56 \mathrm{E}-01 & 1.52 \mathrm{E}-01 & 1.41 \mathrm{E}-01 \\ 4.34 \mathrm{E}-06 & 1.59 \mathrm{E}-05 & 5.53 \mathrm{E}-05 & 1.80 \mathrm{E}-04 & 5.45 \mathrm{E}-04 & 1.51 \mathrm{E}-03 & 3.82 \mathrm{E}-03 & 8.71 \mathrm{E}-03 & 1.79 \mathrm{E}-02 & 3.31 \mathrm{E}-02 & 5.49 \mathrm{E}-02 & 8.26 \mathrm{E}-02 & 1.13 \mathrm{E}-01 & 1.42 \mathrm{E}-01 & 1.65 \mathrm{E}-01 & 1.80 \mathrm{E}-01 & 1.86 \mathrm{E}-01 \\ . & & & \end{array}$

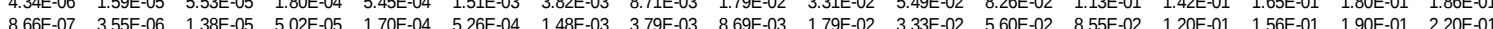
$\begin{array}{lllllllllllllllll}1.55 E-07 & 7.09 E-07 & 3.08 E-06 & 1.25 E-05 & 4.73 E-05 & 1.64 E-04 & 5.17 E-04 & 1.47 E-03 & 3.78 E-03 & 8.71 E-03 & 1.81 E-02 & 3.39 E-02 & 5.79 E-02 & 9.08 E-02 & 1.32 E-01 & 1.80 E-01 & 2.32 E-01\end{array}$

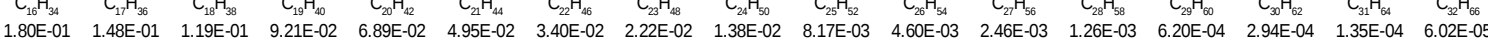

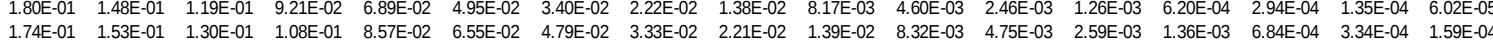

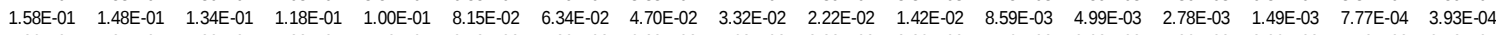

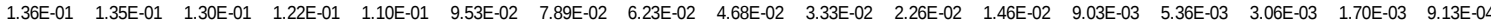

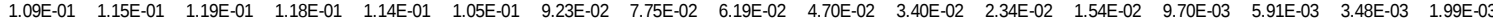

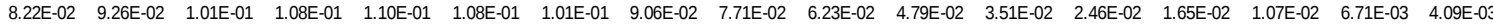

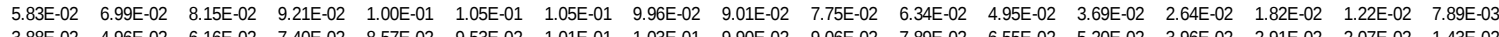

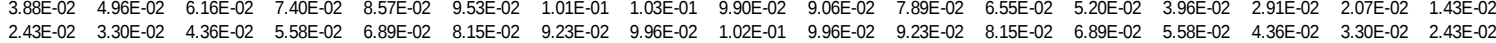

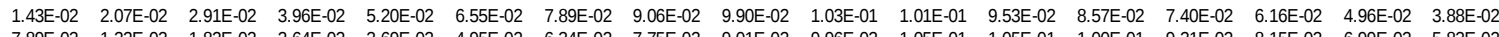

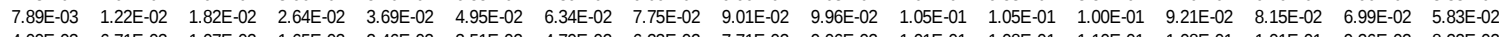

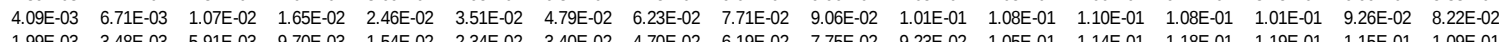

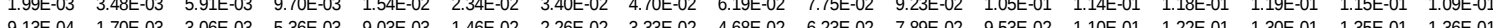

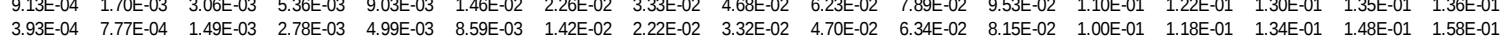

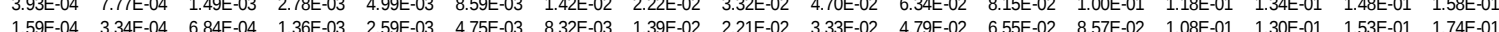

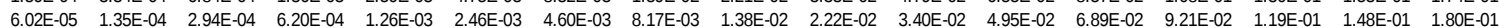

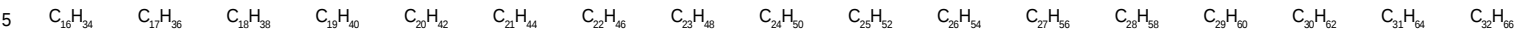

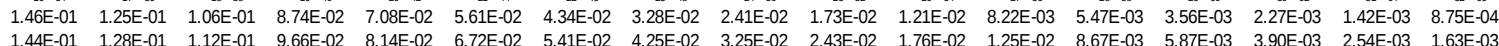

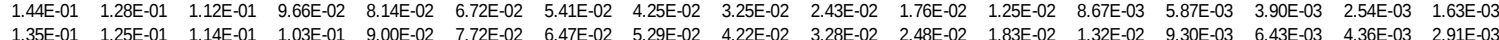

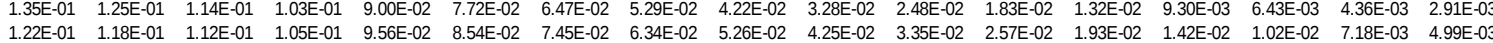

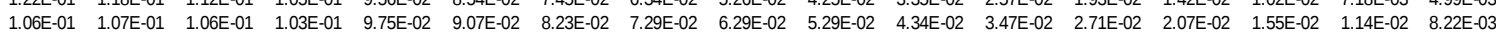

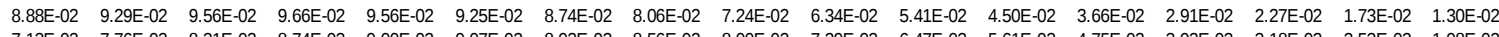

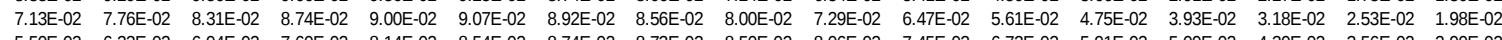

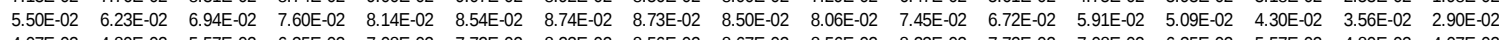

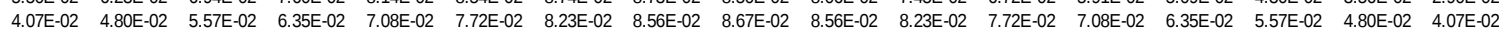

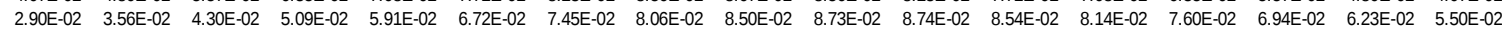

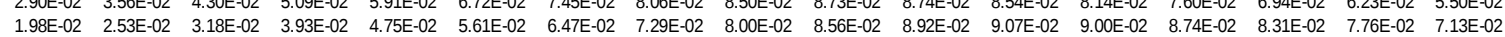

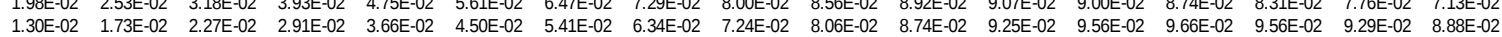

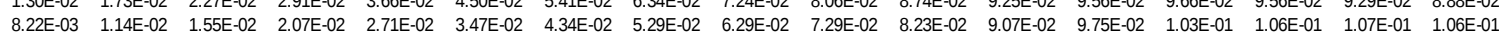
$\begin{array}{lllllllllllllllll}8.22 E-03 & 1.14 E-02 & 1.55 E-02 & 2.07 E-02 & 2.71 E-02 & 3.47 E-02 & 4.34 E-02 & 5.29 E-02 & 6.29 E-02 & 7.25 E-02 & 8.23 E-02 & 9.07 E-02 & 9.75 E-02 & 1.03 E-01 & 1.06 E-01 & 1.07 E-01 & 1.06 E-01 \\ 4.99 E-03 & 7.18 E-03 & 1.02 E-02 & 1.42 E-02 & 1.93 E-02 & 2.57 E-02 & 3.35 E-02 & 4.25 E-02 & 5.26 E-02 & 6.34 E-02 & 7.45 E-02 & 8.54 E-02 & 9.56 E-02 & 1.05 E-01 & 1.12 E-01 & 1.18 E-01 & 1.22 E-01\end{array}$ $\begin{array}{lllllllllllllllll}2.91 E-03 & 4.36 E-03 & 6.43 E-03 & 9.30 E-03 & 1.32 E-02 & 1.83 E-02 & 2.48 E-02 & 3.28 E-02 & 4.22 E-02 & 5.29 E-02 & 6.47 E-02 & 7.72 E-02 & 9.00 E-02 & 1.03 E-01 & 1.14 E-01 & 1.25 E-01 & 1.35 E-01\end{array}$

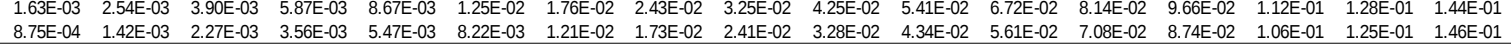


402 Table 1-S. Input modal composition mass fractions (by columns) in the nucleation mode and composition standard deviation for involatile core of $1 \%$.

404

405

406

407

408

409

410

411

412

413

414

415

416

417

418

419

420

421

422

423

424

425

426 


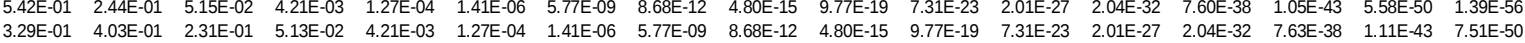

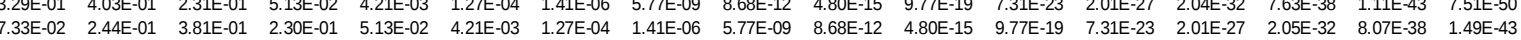

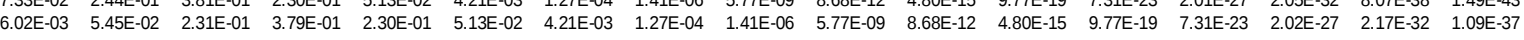
\begin{tabular}{lllllllllllllllll}
$1.82 E-04$ & $4.47 E-03$ & $5.15 E-02$ & $2.30 E-01$ & $3.79 E-01$ & $2.30 E-01$ & $5.13 E-02$ & $4.21 E-03$ & $1.27 E-04$ & $1.41 E-06$ & $5.77 E-09$ & $8.68 E-12$ & $4.80 E-15$ & $9.77 E-19$ & $7.34 E-23$ & $2.14 E-27$ & $2.92 E-32$ \\
\hline
\end{tabular} \begin{tabular}{lllllllllllllllll}
$2.02 E-06$ & $1.35 E-04$ & $4.23 E-03$ & $5.13 E-02$ & $2.30 E-01$ & $3.79 E-01$ & $2.30 E-01$ & $5.13 E-02$ & $4.21 E-03$ & $1.27 E-04$ & $1.41 E-06$ & $5.77 E-09$ & $8.68 E-12$ & $4.80 E-15$ & $9.81 E-19$ & $7.76 E-23$ & $2.88 E-27$ \\
\hline
\end{tabular} \begin{tabular}{llllllllllllllllll}
$8.25 E-09$ & $1.50 E-06$ & $1.28 E-04$ & $4.21 \mathrm{E}-03$ & $5.13 \mathrm{E}-02$ & $2.30 \mathrm{E}-01$ & $3.79 \mathrm{E}-01$ & $2.30 \mathrm{E}-01$ & $5.13 \mathrm{E}-02$ & $4.21 \mathrm{E}-03$ & $1.27 \mathrm{E}-04$ & $1.41 \mathrm{E}-06$ & $5.77 \mathrm{E}-09$ & $8.68 \mathrm{E}-12$ & $4.82 \mathrm{E}-15$ & $1.04 \mathrm{E}-18$ & $1.05 \mathrm{E}-22$ \\
\hline
\end{tabular}

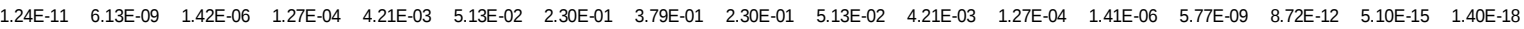

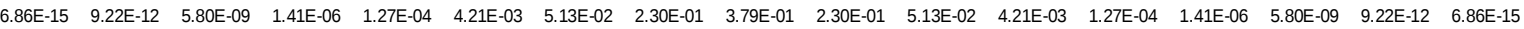

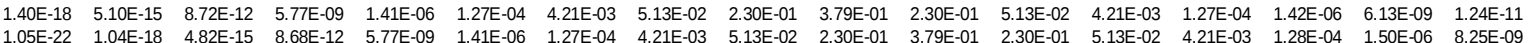

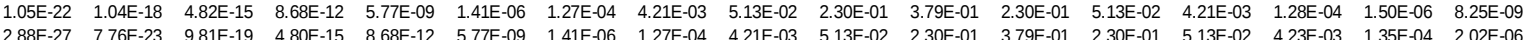

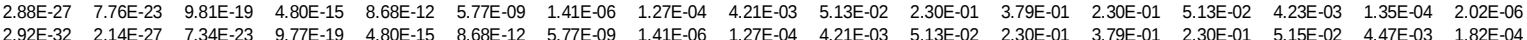

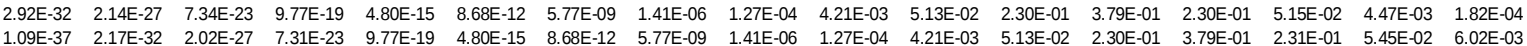

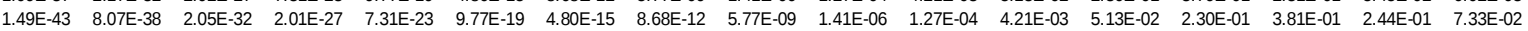

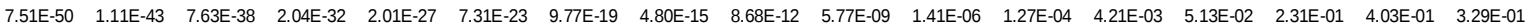

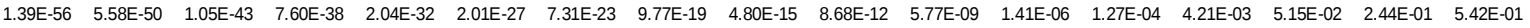

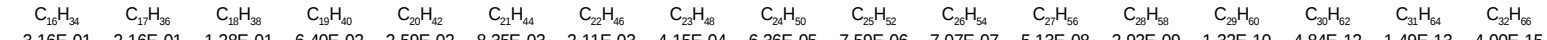

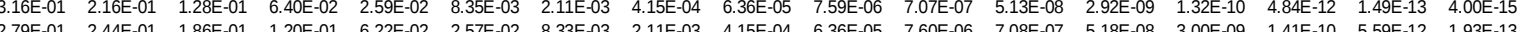

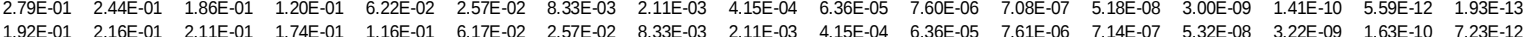

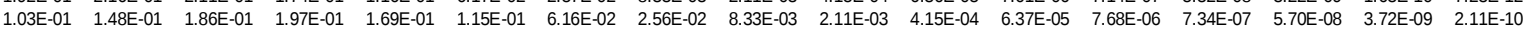

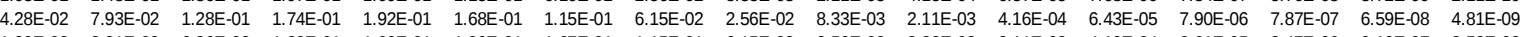

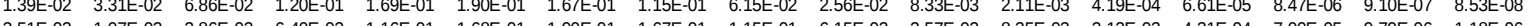

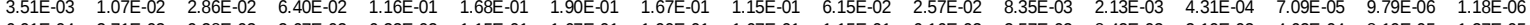

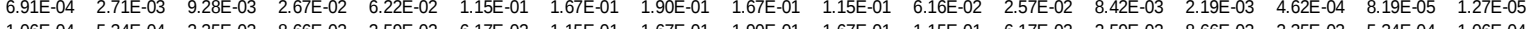

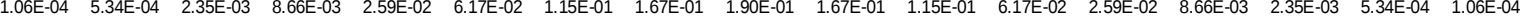

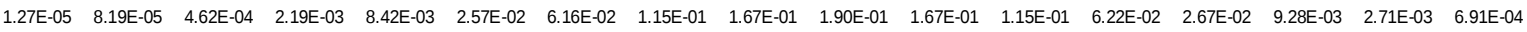
\begin{tabular}{lllllllllllllllll}
$1.18 E-06$ & $9.79 E-06$ & $7.09 E-05$ & $4.31 \mathrm{E}-04$ & $2.13 \mathrm{E}-03$ & $8.35 \mathrm{E}-03$ & $2.57 \mathrm{E}-02$ & $6.15 \mathrm{E}-02$ & $1.15 \mathrm{E}-01$ & $1.67 \mathrm{E}-01$ & $1.90 \mathrm{E}-01$ & $1.68 \mathrm{E}-01$ & $1.16 \mathrm{E}-01$ & $6.40 \mathrm{E}-02$ & $2.86 \mathrm{E}-02$ & $1.07 \mathrm{E}-02$ & $3.51 \mathrm{E}-03$ \\
\hline
\end{tabular}

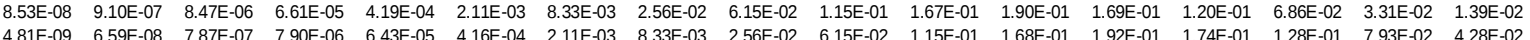

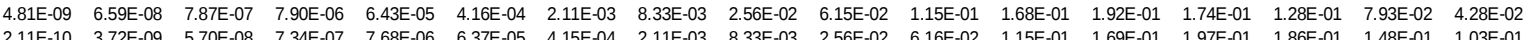
\begin{tabular}{lllllllllllllllll}
$2.11 E-10$ & $3.72 E-09$ & $5.70 E-08$ & $7.34 E-07$ & $7.68 E-06$ & $6.37 E-05$ & $4.15 E-04$ & $2.11 E-03$ & $8.33 E-03$ & $2.56 E-02$ & $6.16 E-02$ & $1.15 E-01$ & $1.69 E-01$ & $1.97 E-01$ & $1.86 E-01$ & $1.48 E-01$ & $1.03 E-01$ \\
\hline
\end{tabular}

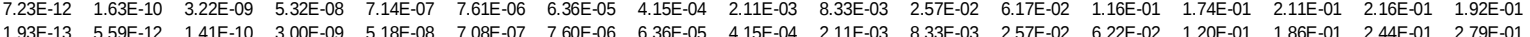

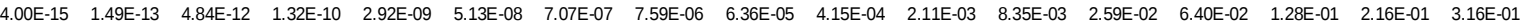

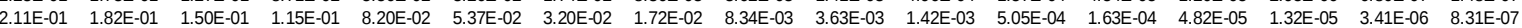

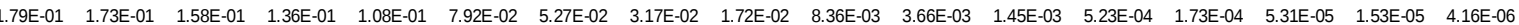

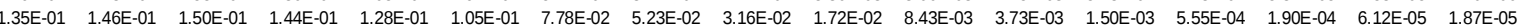

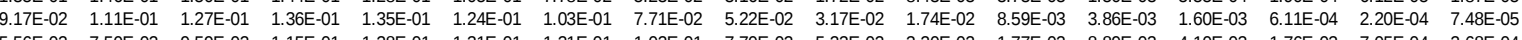

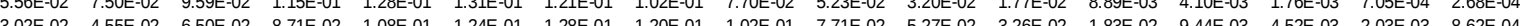

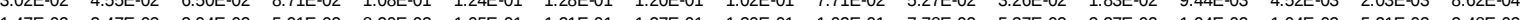

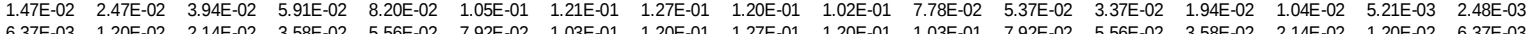

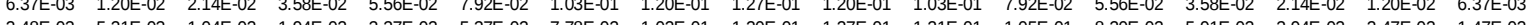

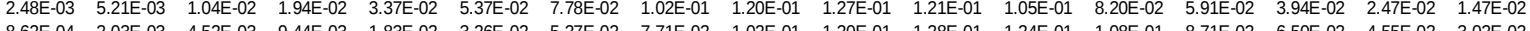
$\begin{array}{lllllllllllllllll}8.62 E-04 & 2.03 E-03 & 4.52 E-03 & 9.44 E-03 & 1.83 E-02 & 3.26 E-02 & 5.27 E-02 & 7.71 E-02 & 1.02 E-01 & 1.20 E-01 & 1.28 E-01 & 1.24 E-01 & 1.08 E-01 & 8.71 E-02 & 6.50 E-02 & 4.55 E-02 & 3.02 E-02\end{array}$

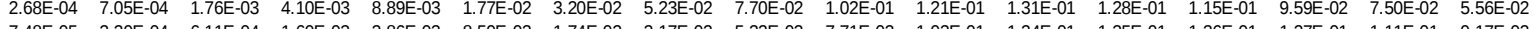
$\begin{array}{lllllllllllllllll}7.48 E-05 & 2.20 E-04 & 6.11 E-04 & 1.60 E-03 & 3.86 E-03 & 8.59 E-03 & 1.74 E-02 & 3.17 E-02 & 5.22 E-02 & 7.71 E-02 & 1.03 E-01 & 1.24 E-01 & 1.35 E-01 & 1.36 E-01 & 1.27 E-01 & 1.11 E-01 & 9.17 E-02\end{array}$

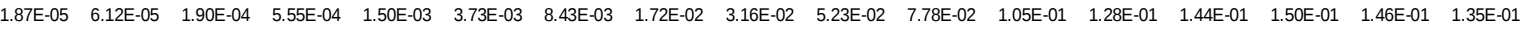

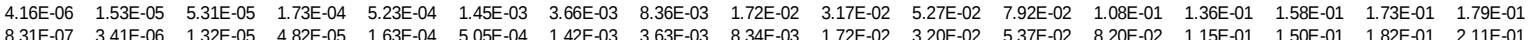

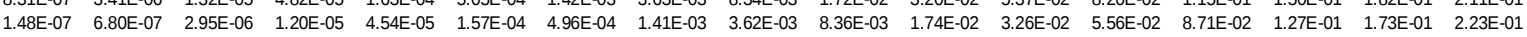

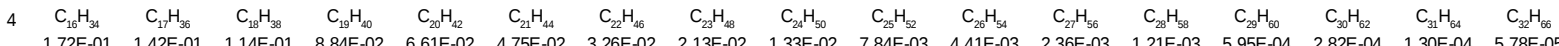

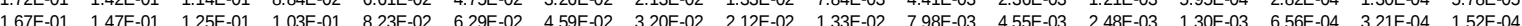

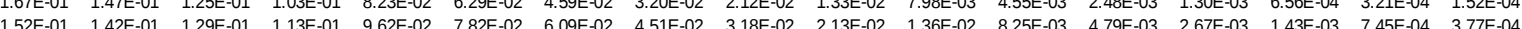
$\begin{array}{llllllllllll}1.52 E & 01 & 142 E & 01\end{array}$

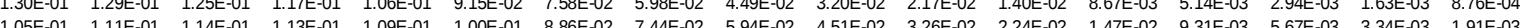

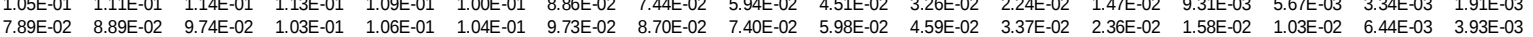

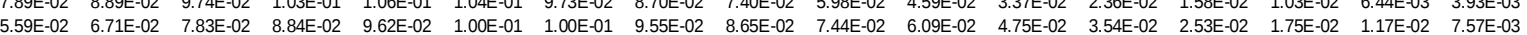

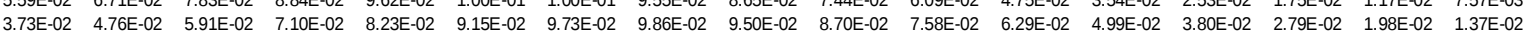

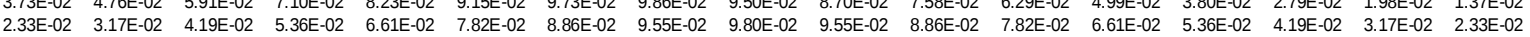

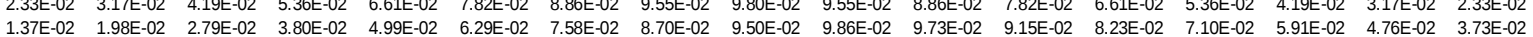

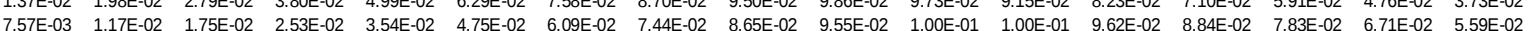

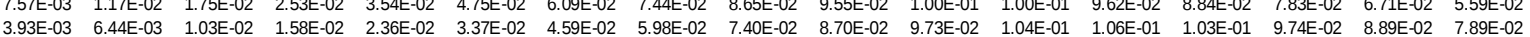

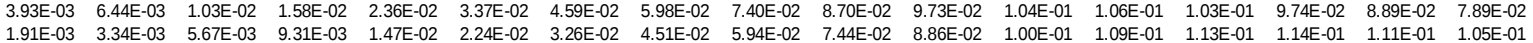

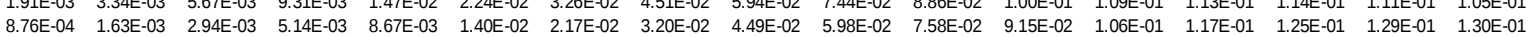

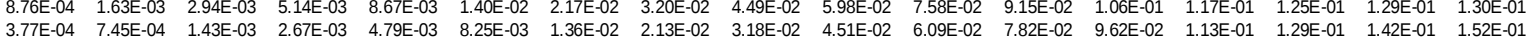
$\begin{array}{lllllllllllllllll}1.52 E-04 & 3.21 E-04 & 6.56 E-04 & 1.30 E-03 & 2.48 E-03 & 4.55 E-03 & 7.98 E-03 & 1.33 E-02 & 2.12 E-02 & 3.20 E-02 & 4.59 E-02 & 6.29 E-02 & 8.23 E-02 & 1.03 E-01 & 1.25 E-01 & 1.47 E-01 & 1.67 E-01\end{array}$

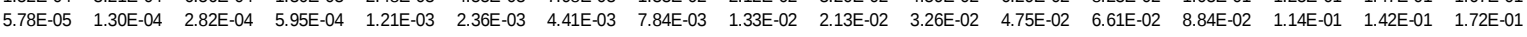

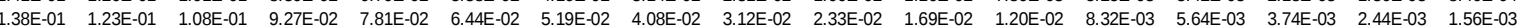

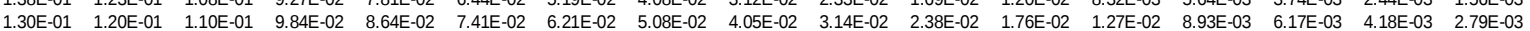

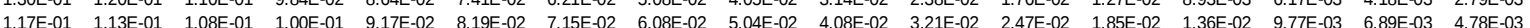

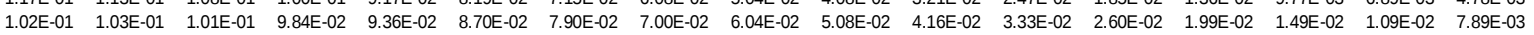

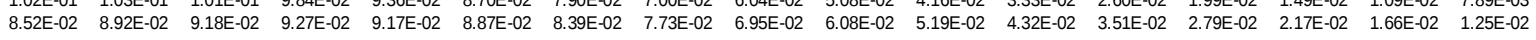

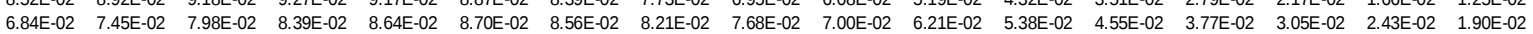

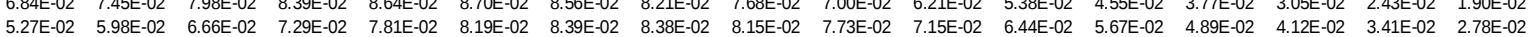

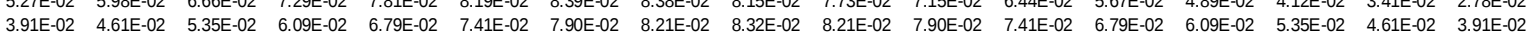

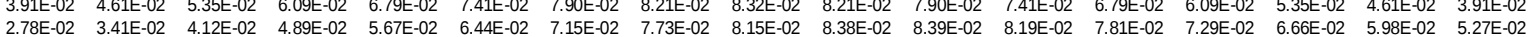

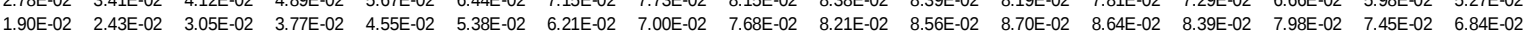

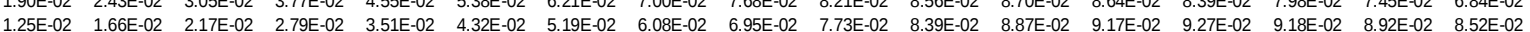

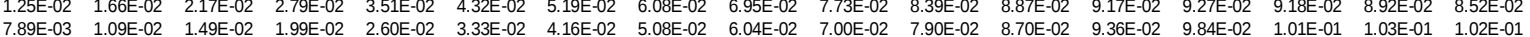

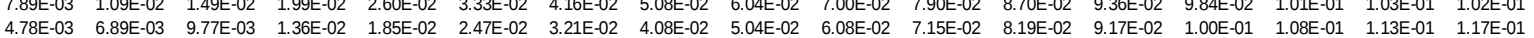

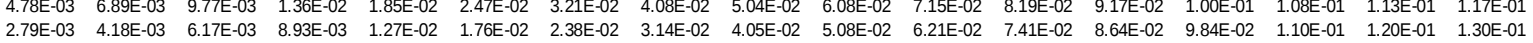

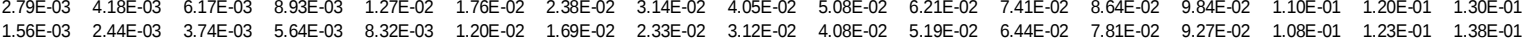

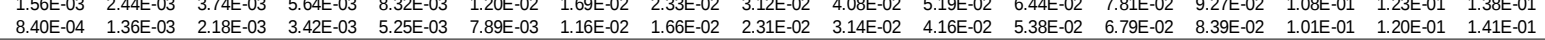


453 Table 2-S. Input modal composition mass fractions (by columns) in the nucleation mode and 454 composition standard deviation for involatile core of $5 \%$.

455

456

457

458

459

460

461

462

463

464

465

466

467

468

469

470

471

472

473

474

475

476

477

478 


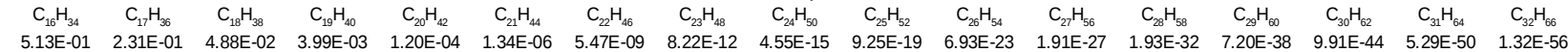

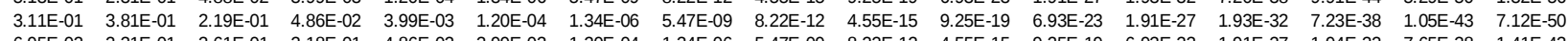

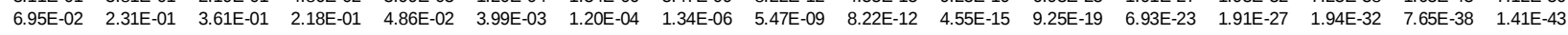

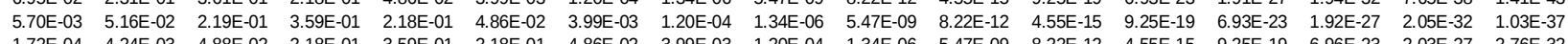

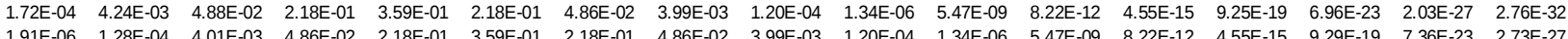
\begin{tabular}{lllllllllllllllll}
$7.82 \mathrm{E}-09$ & $1.42 \mathrm{E}-06$ & $1.21 \mathrm{E}-04$ & $3.99 \mathrm{E}-03$ & $4.86 \mathrm{E}-02$ & $2.18 \mathrm{E}-01$ & $3.59 \mathrm{E}-01$ & $2.18 \mathrm{E}-01$ & $4.86 \mathrm{E}-02$ & $3.99 \mathrm{E}-03$ & $1.20 \mathrm{E}-04$ & $1.34 \mathrm{E}-06$ & $5.47 \mathrm{E}-09$ & $8.22 \mathrm{E}-12$ & $4.57 \mathrm{E}-15$ & $9.83 \mathrm{E}-19$ & $9.90 \mathrm{E}-23$ \\
\hline
\end{tabular}

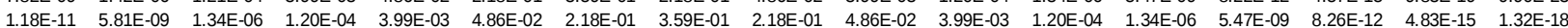

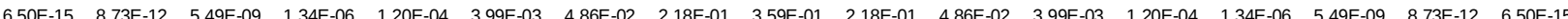

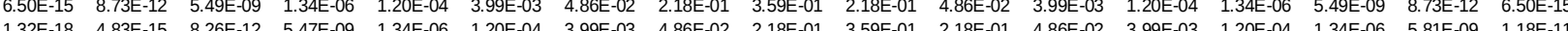

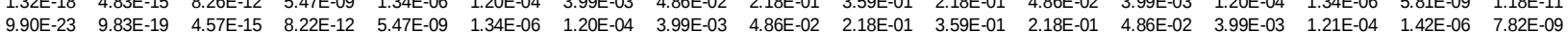

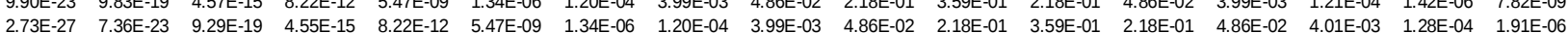

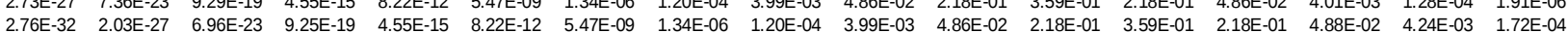

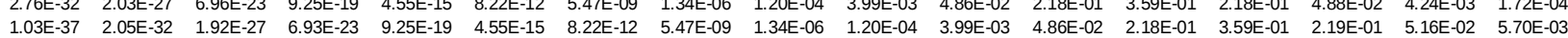

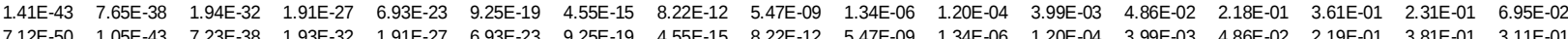

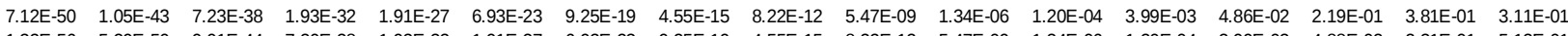

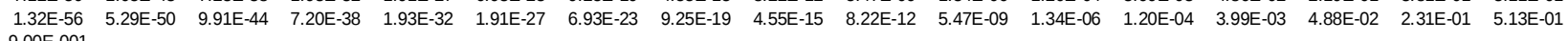

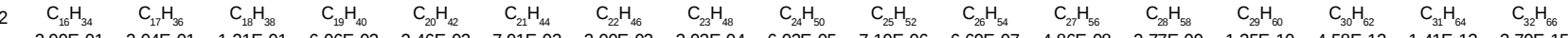

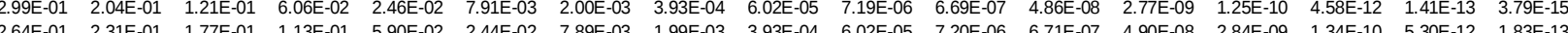
$\begin{array}{llllllllllll}2.64 \mathrm{E}-01 & 2.31 \mathrm{E}-01 & 1.77 \mathrm{E}-01 & 1.13 \mathrm{E}-01 & 5.90 \mathrm{E}-02 & 2.44 \mathrm{E}-02 & 7.89 \mathrm{E}-03 & 1.99 \mathrm{E}-03 & 3.93 \mathrm{E}-04 & 6.02 \mathrm{E}-05 & 7 & \end{array}$

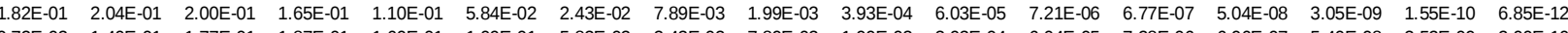

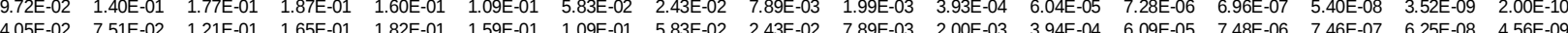

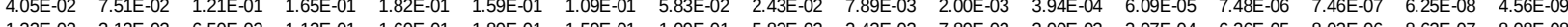

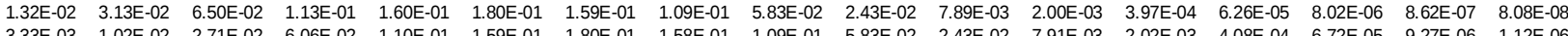
$\begin{array}{lllllllllllllllll}3.33 \mathrm{E}-03 & 1.02 \mathrm{E}-02 & 2.71 \mathrm{E}-02 & 6.06 \mathrm{E}-02 & 1.10 \mathrm{E}-01 & 1.59 \mathrm{E}-01 & 1.80 \mathrm{E}-01 & 1.58 \mathrm{E}-01 & 1.09 \mathrm{E}-01 & 5.83 \mathrm{E}-02 & 2.43 \mathrm{E}-02 & 7.91 \mathrm{E}-03 & 2.02 \mathrm{E}-03 & 4.08 \mathrm{E}-04 & 6.72 \mathrm{E}-05 & 9.27 \mathrm{E}-06 & 1.12 \mathrm{E}-06 \\ 6.55 \mathrm{E}-04 & 2.57 \mathrm{E}-03 & 8.80 \mathrm{E}-03 & 2.53 \mathrm{E}-02 & 5.90 \mathrm{E}-02 & 1.09 \mathrm{E}-01 & 1.59 \mathrm{E}-01 & 1.80 \mathrm{E}-01 & 1.58 \mathrm{E}-01 & 1.09 \mathrm{E}-01 & 5.83 \mathrm{E}-02 & 2.44 \mathrm{E}-02 & 7.98 \mathrm{E}-03 & 2.07 \mathrm{E}-03 & 4.38 \mathrm{E}-04 & 7.76 \mathrm{E}-05 & 1.20 \mathrm{E}-05 \\ 1 & & & \end{array}$

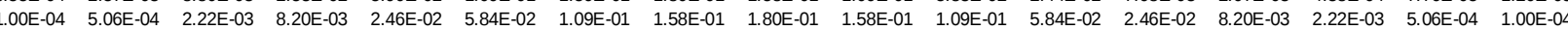

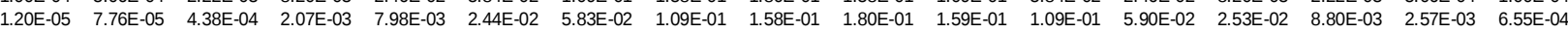

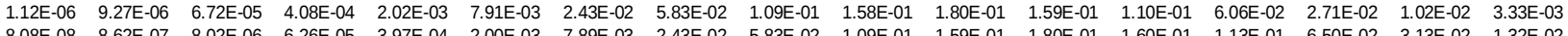
\begin{tabular}{lllllllllllllllll}
$8.08 \mathrm{E}-08$ & $8.62 \mathrm{E}-07$ & $8.02 \mathrm{E}-06$ & $6.26 \mathrm{E}-05$ & $3.97 \mathrm{E}-04$ & $2.00 \mathrm{E}-03$ & $7.89 \mathrm{E}-03$ & $2.43 \mathrm{E}-02$ & $5.83 \mathrm{E}-02$ & $1.09 \mathrm{E}-01$ & $1.59 \mathrm{E}-01$ & $1.80 \mathrm{E}-01$ & $1.60 \mathrm{E}-01$ & $1.13 \mathrm{E}-01$ & $6.50 \mathrm{E}-02$ & $3.13 \mathrm{E}-02$ & $1.32 \mathrm{E}-02$ \\
\hline
\end{tabular}

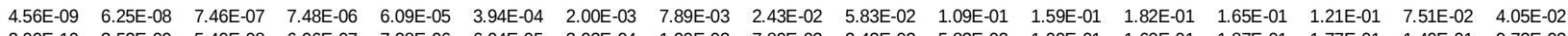

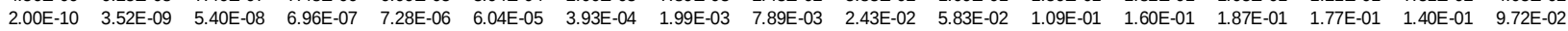

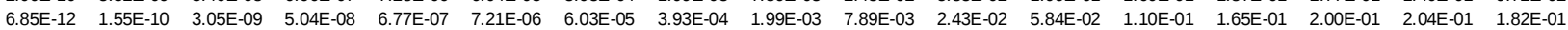

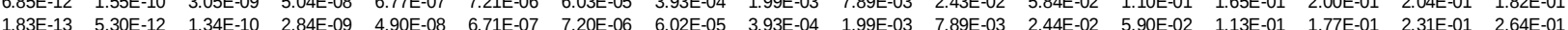

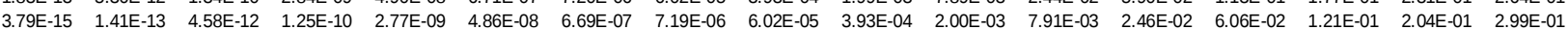

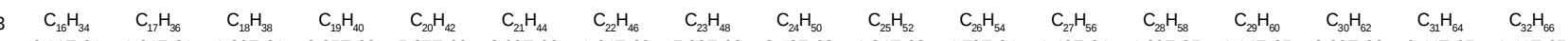

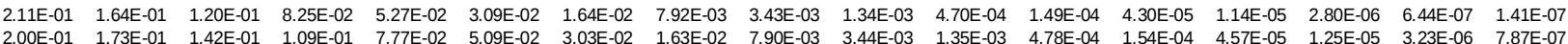

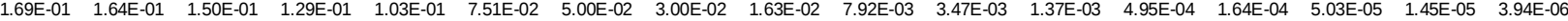

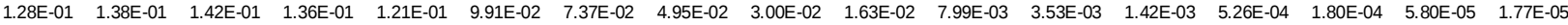

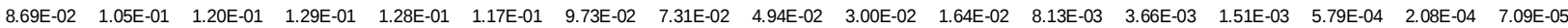

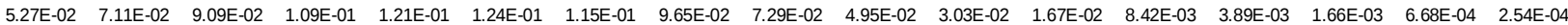

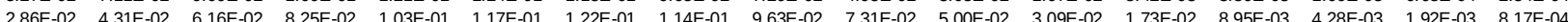

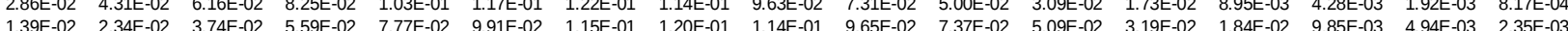

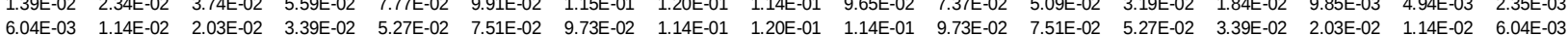

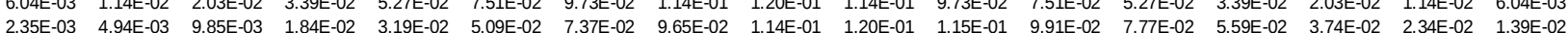

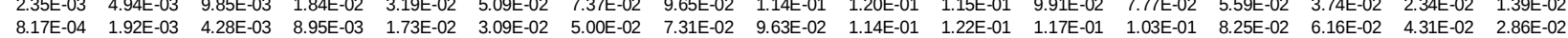

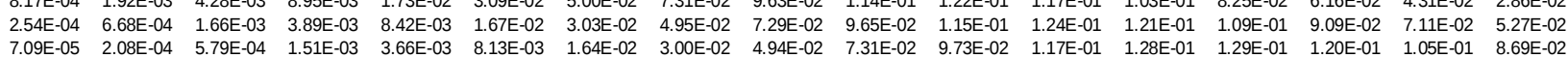

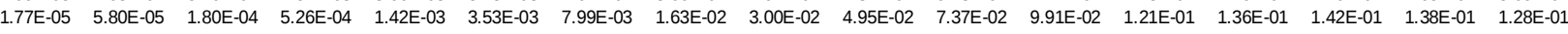

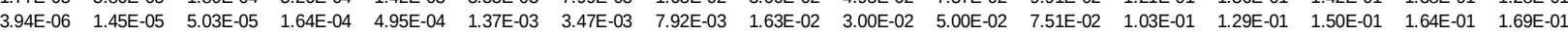

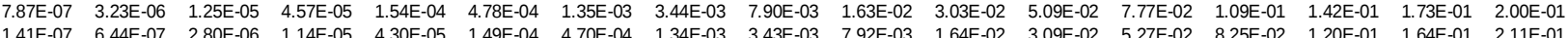

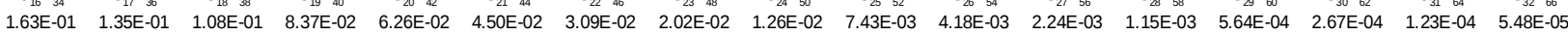
\begin{tabular}{lllllllllllllllll}
$1.58 E-01$ & $1.39 E-01$ & $1.18 E-01$ & $9.79 E-02$ & $7.79 E-02$ & $5.96 E-02$ & $4.35 E-02$ & $3.03 E-02$ & $2.01 E-02$ & $1.26 E-02$ & $7.56 E-03$ & $4.31 E-03$ & $2.35 E-03$ & $1.23 E-03$ & $6.22 E-04$ & $3.04 E-04$ & $1.44 E-04$ \\
\hline
\end{tabular}

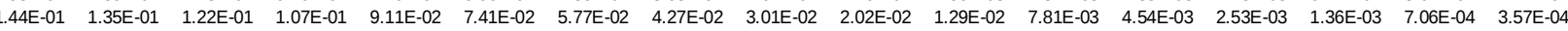

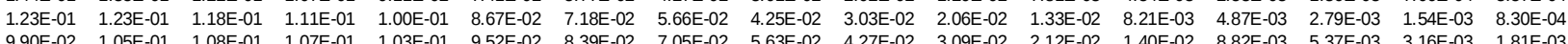
\begin{tabular}{lllllllllllllllll}
$9.90 \mathrm{E}-02$ & $1.05 \mathrm{E}-01$ & $1.08 \mathrm{E}-01$ & $1.07 \mathrm{E}-01$ & $1.03 \mathrm{E}-01$ & $9.52 \mathrm{E}-02$ & $8.39 \mathrm{E}-02$ & $7.05 \mathrm{E}-02$ & $5.63 \mathrm{E}-02$ & $4.27 \mathrm{E}-02$ & $3.09 \mathrm{E}-02$ & $2.12 \mathrm{E}-02$ & $1.40 \mathrm{E}-02$ & $8.82 \mathrm{E}-03$ & $5.37 \mathrm{E}-03$ & $3.16 \mathrm{E}-03$ & $1.81 \mathrm{E}-03$ \\
\hline
\end{tabular}

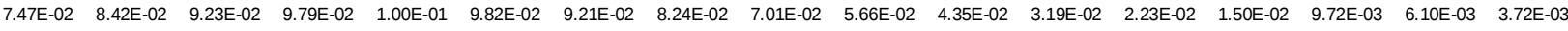

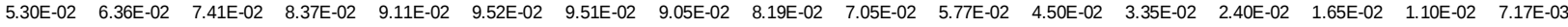

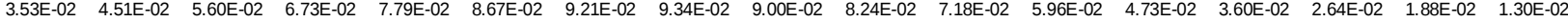

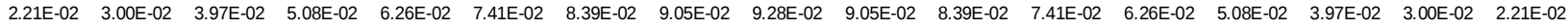

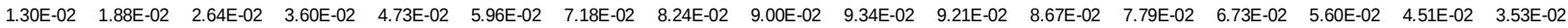

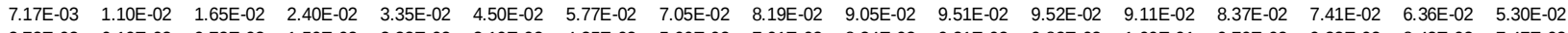

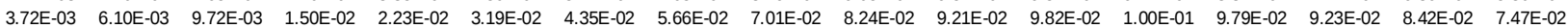

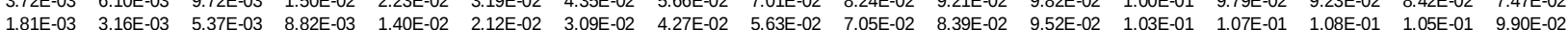

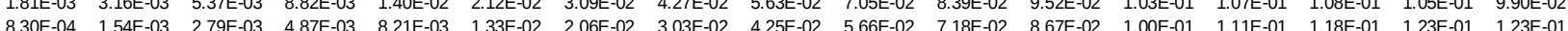
$\begin{array}{lllllllllllllllll}8.30 \mathrm{E}-04 & 1.54 \mathrm{E}-03 & 2.79 \mathrm{E}-03 & 4.87 \mathrm{E}-03 & 8.21 \mathrm{E}-03 & 1.33 \mathrm{E}-02 & 2.06 \mathrm{E}-02 & 3.03 \mathrm{E}-02 & 4.25 \mathrm{E}-02 & 5.66 \mathrm{E}-02 & 7.18 \mathrm{E}-02 & 8.67 \mathrm{E}-02 & 1.00 \mathrm{E}-01 & 1.11 \mathrm{E}-01 & 1.18 \mathrm{E}-01 & 1.23 \mathrm{E}-01 & 1.23 \mathrm{E}-01 \\ 3.57 \mathrm{E}-04 & 7.06 \mathrm{E}-04 & 1.36 \mathrm{E}-03 & 2.53 \mathrm{E}-03 & 4.54 \mathrm{E}-03 & 7.81 \mathrm{E}-03 & 1.29 \mathrm{E}-02 & 2.02 \mathrm{E}-02 & 3.01 \mathrm{E}-02 & 4.27 \mathrm{E}-02 & 5.77 \mathrm{E}-02 & 7.41 \mathrm{E}-02 & 9.11 \mathrm{E}-02 & 1.07 \mathrm{E}-01 & 1.22 \mathrm{E}-01 & 1.35 \mathrm{E}-01 & 1.44 \mathrm{E}-01 \\ 1.45 & & & \end{array}$

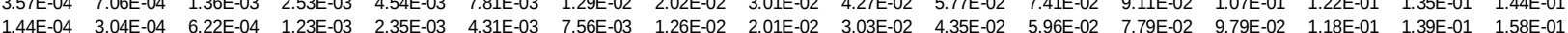

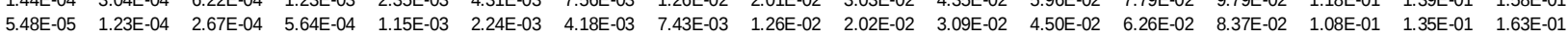

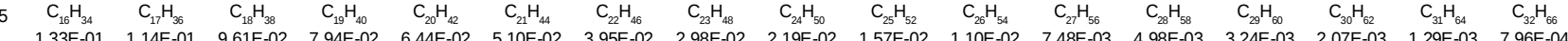

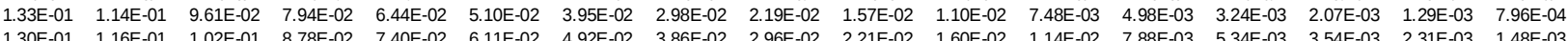

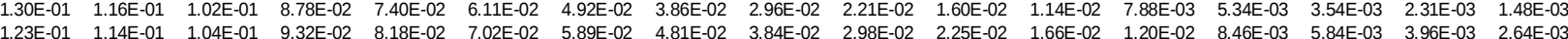

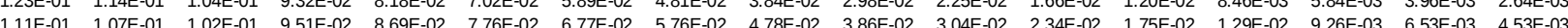

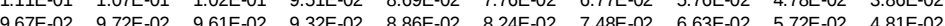
$1.41 \mathrm{E}-02 \quad 1.03 \mathrm{E}-02 \quad 7.47 \mathrm{E}-03$

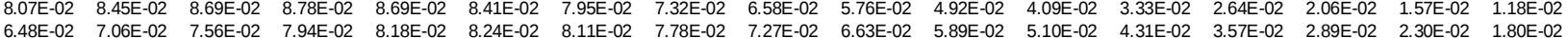

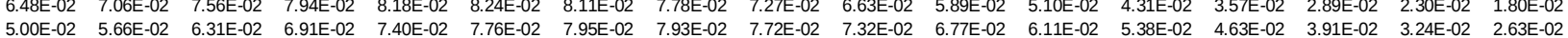

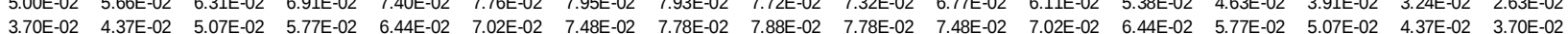

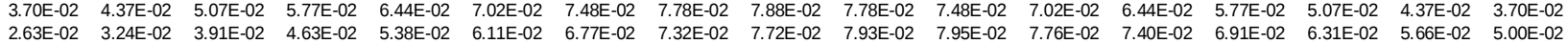

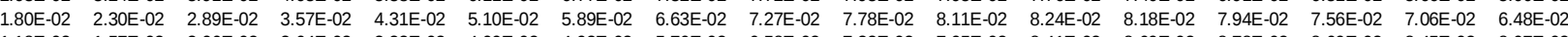

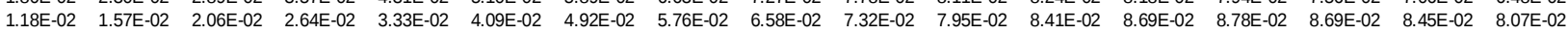

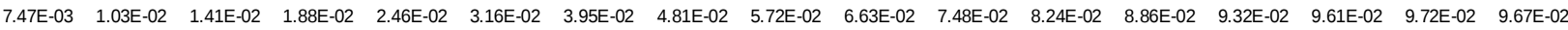

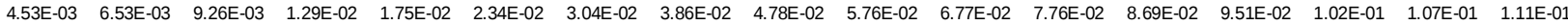

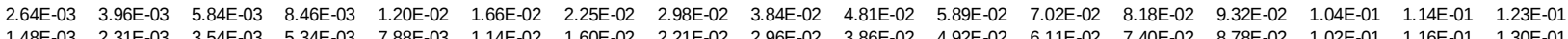

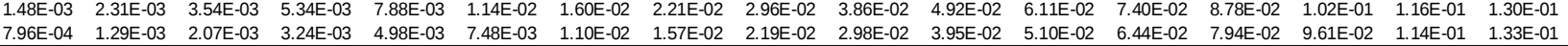


506 Table 3-S. Input modal composition mass fractions (by columns) in the nucleation mode and

507 composition standard deviation for involatile core of $10 \%$.

508

509

510

511

512

513

514

515

516

517

518

519

520

521

522

523

524

525

526

527

528

529

530 


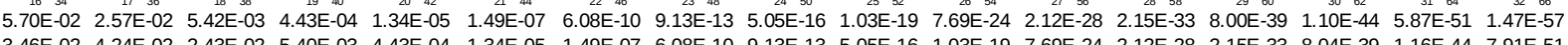

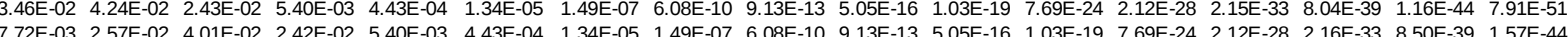

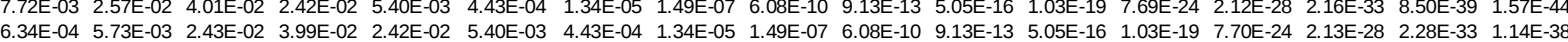

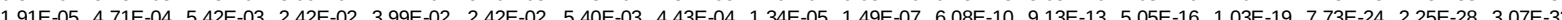

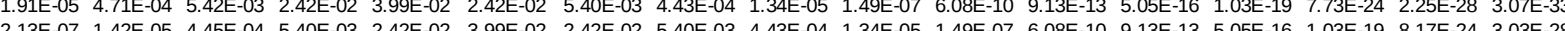

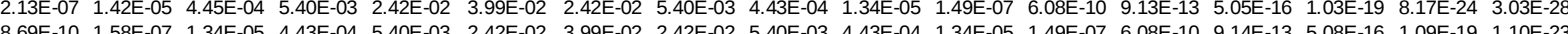
8.69E-10 1.5EE 07 1.34E

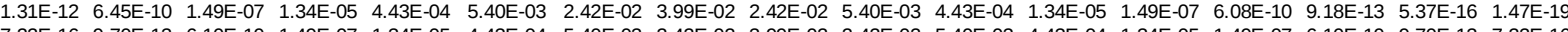

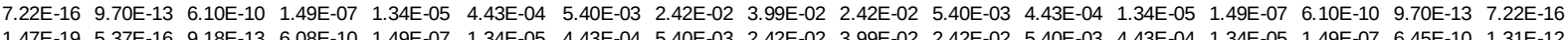

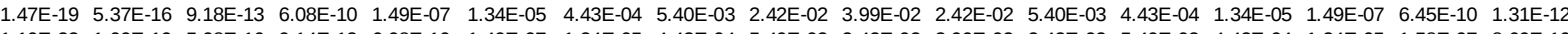

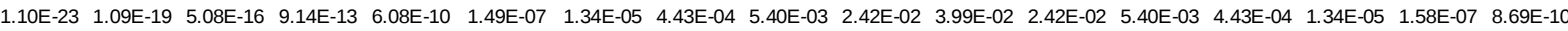

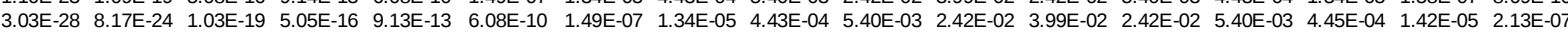

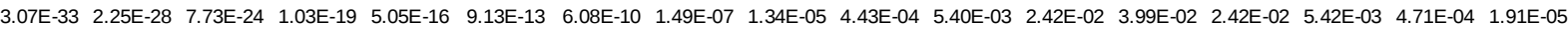

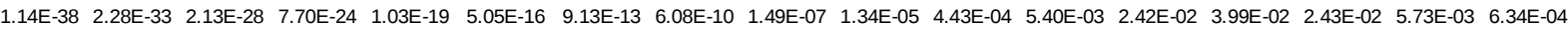

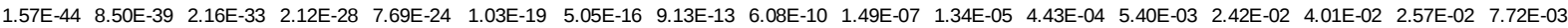

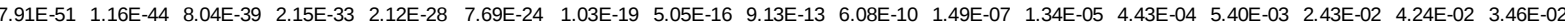

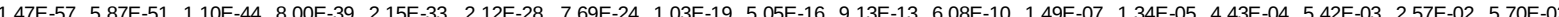

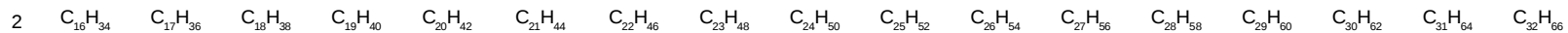

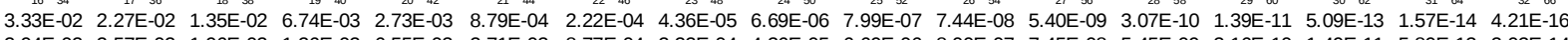

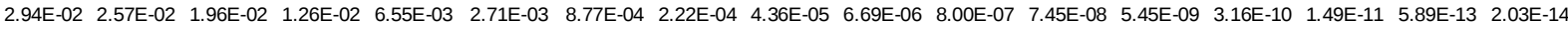

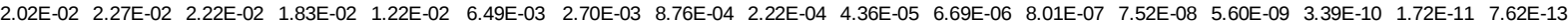

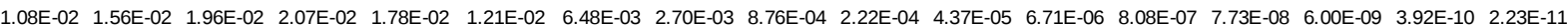

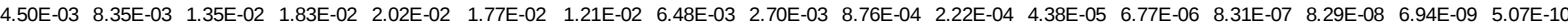

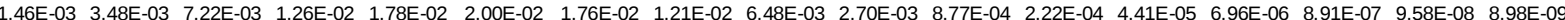

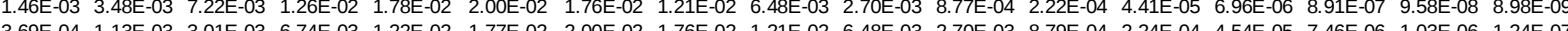

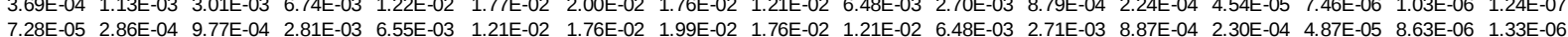

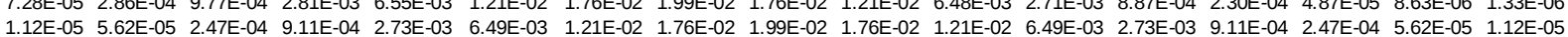

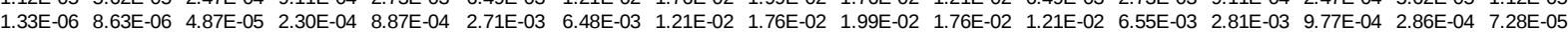

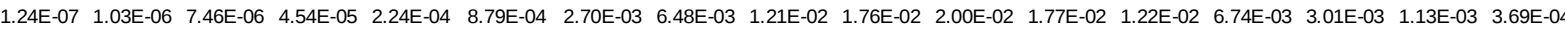

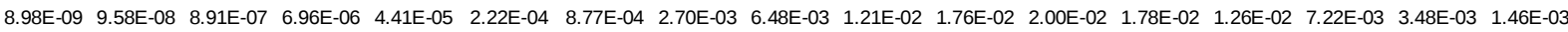

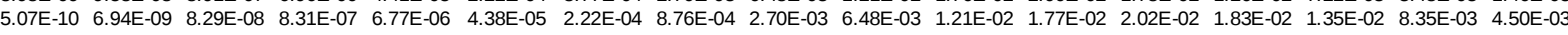

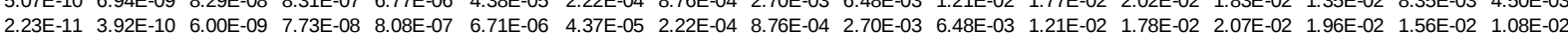

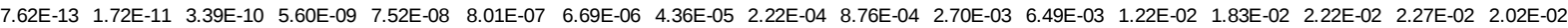

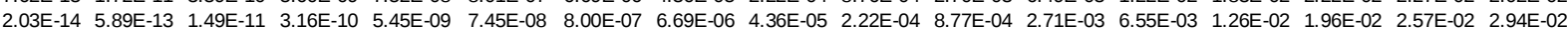

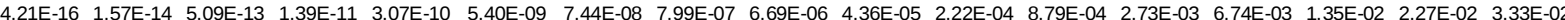

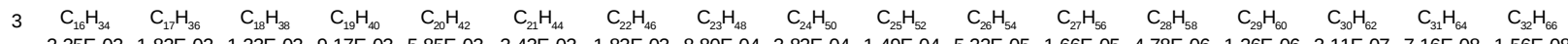

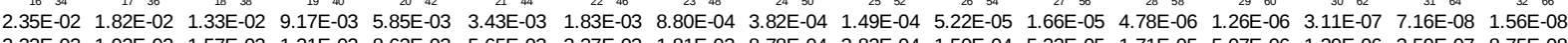

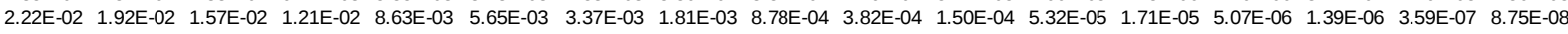

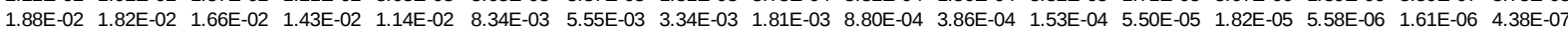

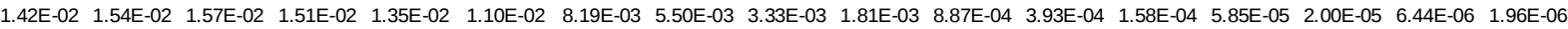

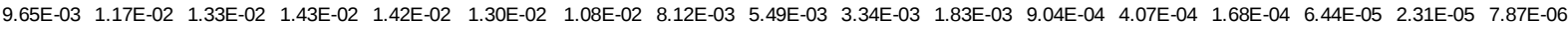

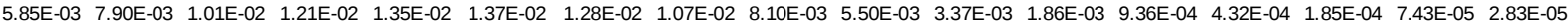
$\begin{array}{lllllllllllllllll}3.18 \mathrm{E}-03 & 4.79 \mathrm{E}-03 & 6.84 \mathrm{E}-03 & 9.17 \mathrm{E}-03 & 1.14 \mathrm{E}-02 & 1.30 \mathrm{E}-02 & 1.35 \mathrm{E}-02 & 1.27 \mathrm{E}-02 & 1.07 \mathrm{E}-02 & 8.12 \mathrm{E}-03 & 5.55 \mathrm{E}-03 & 3.43 \mathrm{E}-03 & 1.36 \mathrm{E}-03 & 9.94 \mathrm{E}-04 & 4.76 \mathrm{E}-04 & 213 \mathrm{E}-04 & 9.08 \mathrm{E}-05\end{array}$

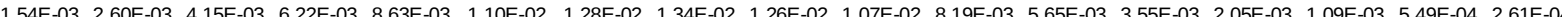
\begin{tabular}{lllllllllllllllllllll}
$1.54 E-03$ & $2.60 E-03$ & $4.15 E-03$ & $6.22 E-03$ & $8.63 E-03$ & $1.10 E-02$ & $1.28 E-02$ & $1.34 E-02$ & $1.26 E-02$ & $1.07 E-02$ & $8.19 E-03$ & $5.65 E-03$ & $3.55 E-03$ & $2.05 E-03$ & $1.09 E-03$ & $5.49 E-04$ & $2.61 E-04$ \\
\hline
\end{tabular}

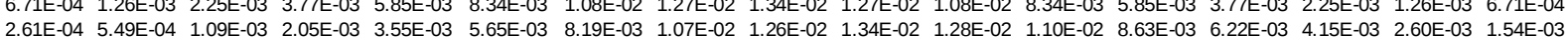

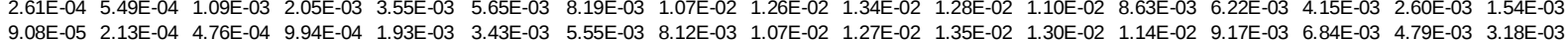

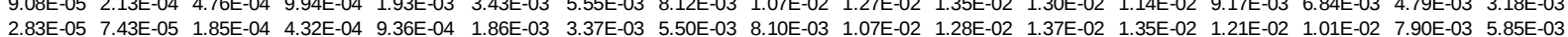

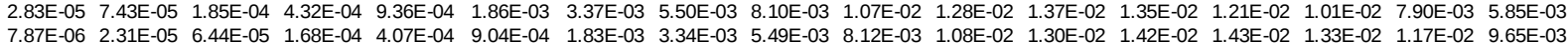

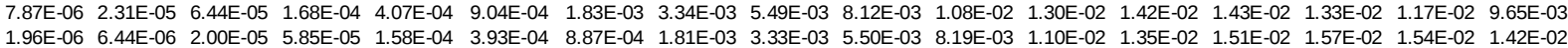

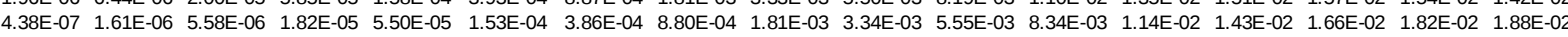

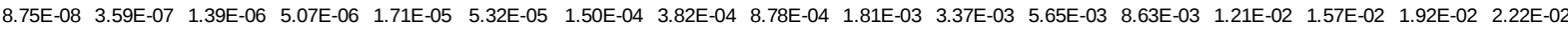

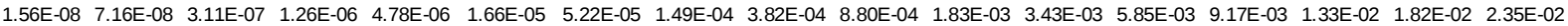

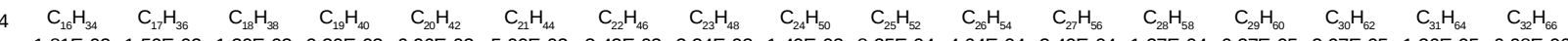

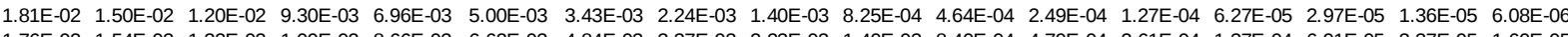

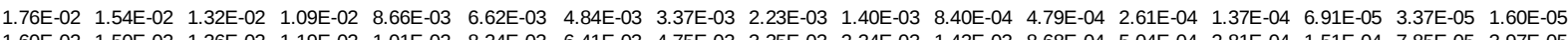

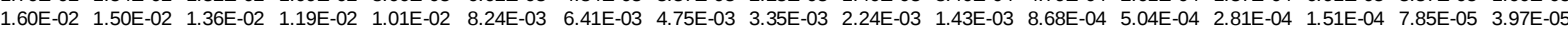

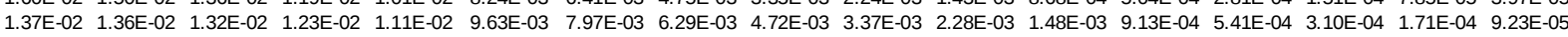

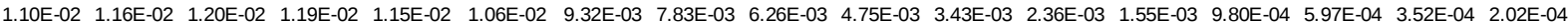

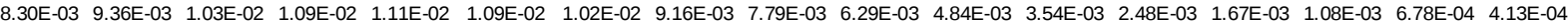

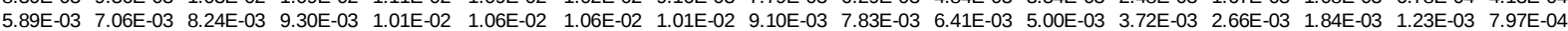

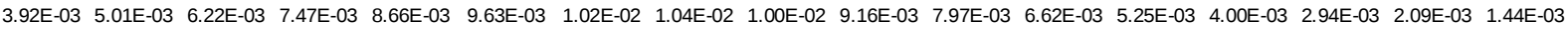

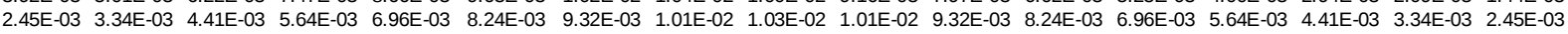

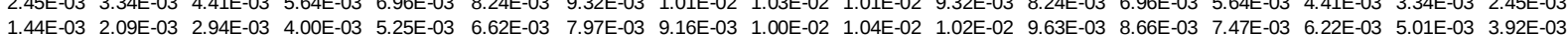

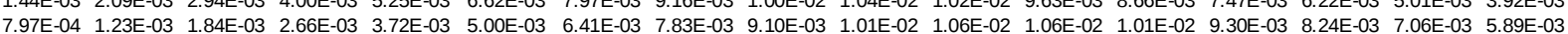

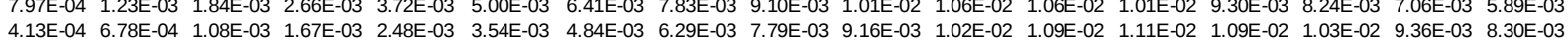

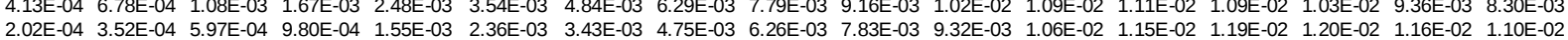

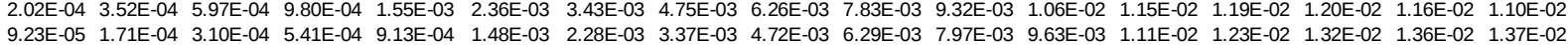

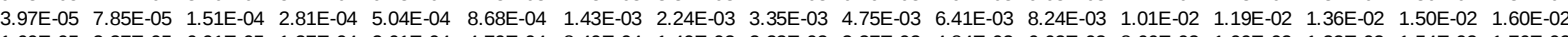

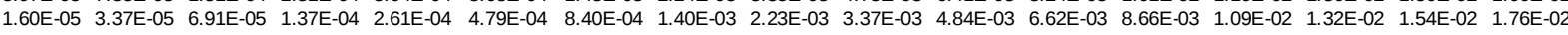

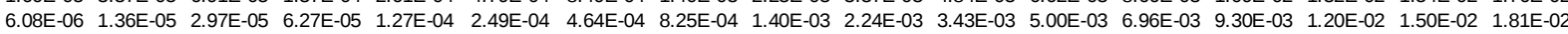

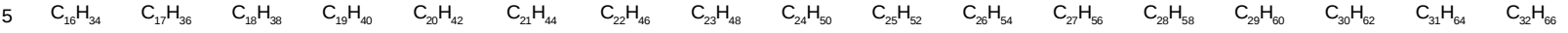

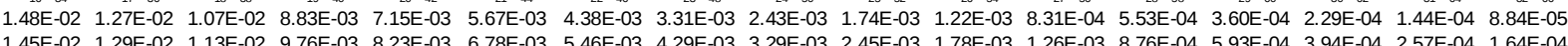

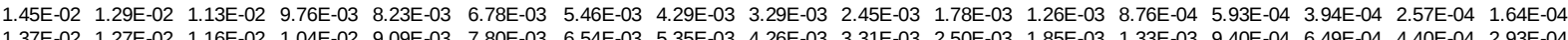

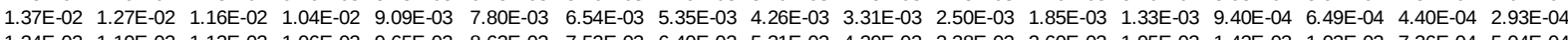

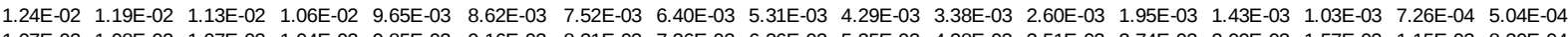

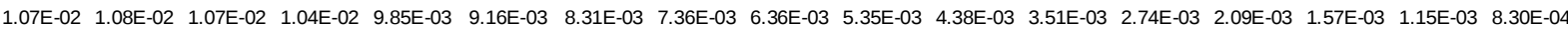

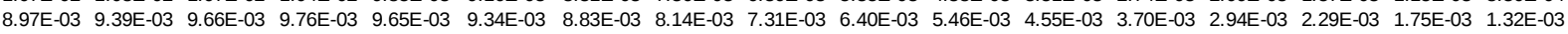

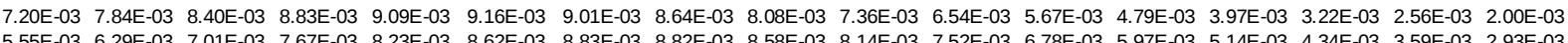
\begin{tabular}{lllllllllllllllllllll}
$5.55 \mathrm{E}-03$ & $6.29 \mathrm{E}-03$ & $7.01 \mathrm{E}-03$ & $7.67 \mathrm{E}-03$ & $8.23 \mathrm{E}-03$ & $8.62 \mathrm{E}-03$ & $8.83 \mathrm{E}-03$ & $8.82 \mathrm{E}-03$ & $8.58 \mathrm{E}-03$ & $8.14 \mathrm{E}-03$ & $7.52 \mathrm{E}-03$ & $6.78 \mathrm{E}-03$ & $5.97 \mathrm{E}-03$ & $5.14 \mathrm{E}-03$ & $4.34 \mathrm{E}-03$ & $3.59 \mathrm{E}-03$ & $2.93 \mathrm{E}-03$ \\
\hline
\end{tabular}

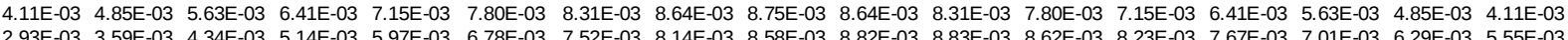

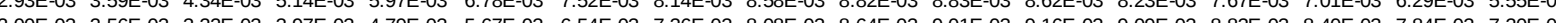

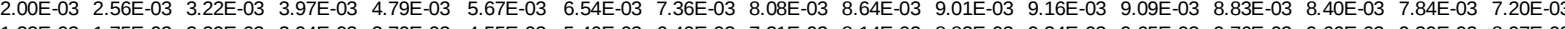

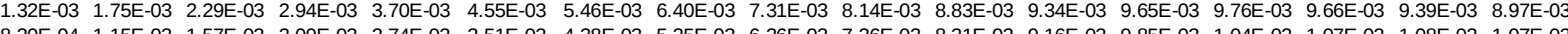

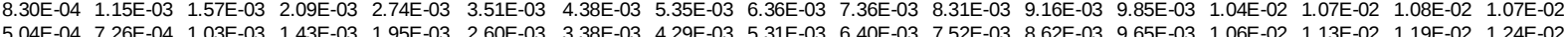

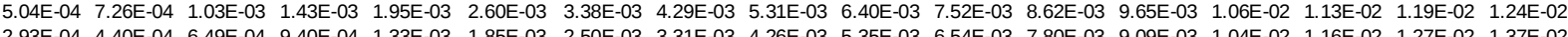

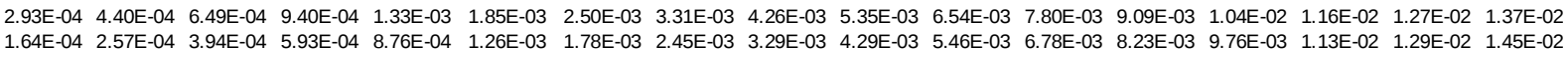

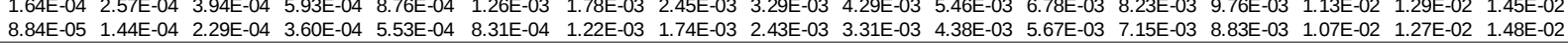


558 Table 4-S. Input modal composition mass fractions (by columns) in the Aitken mode and 559 composition standard deviation for involatile core of $90 \%$.

560

561

562

563

564

565

566

567

568

569

570

571

572

573

574

575

576

577

578

579

580

581

582 
584

$\begin{array}{ccc} & \text { n-alkanes } & \text { Chickos\&Lipkind } \\ 585 & \mathrm{C}_{\mathrm{n}} \mathrm{H}_{(2 n+2)} & 2008 \\ & n & \\ & 16 & \\ 586 & 17 & \\ & 18 & \\ 587 & 19 & \\ & 20 & \\ 588 & 21 & 6.93 \mathrm{E}-004 \\ & 22 & 2.09 \mathrm{E}-004 \\ & 23 & 6.95 \mathrm{E}-005 \\ 589 & 24 & 2.31 \mathrm{E}-005 \\ & 25 & 8.51 \mathrm{E}-006 \\ 590 & 26 & 2.83 \mathrm{E}-006 \\ & 27 & 1.15 \mathrm{E}-006 \\ & 28 & 3.14 \mathrm{E}-007 \\ 591 & 29 & 1.05 \mathrm{E}-007 \\ & 30 & 3.48 \mathrm{E}-008 \\ 592 & 31 & 1.16 \mathrm{E}-008 \\ & 32 & 3.49 \mathrm{E}-009\end{array}$

EPI Suite

9.29E-001

4.32E-001

$1.95 \mathrm{E}-001$

$8.97 \mathrm{E}-002$

4.13E-002

$1.89 \mathrm{E}-002$

$9.23 \mathrm{E}-003$

4.73E- -003

2.26E-003

$1.28 \mathrm{E}-002$

$7.02 \mathrm{E}-004$

$1.31 \mathrm{E}-004$

$2.06 \mathrm{E}-004$

$1.27 \mathrm{E}-004$

$7.49 \mathrm{E}-005$

4.54E-005

$2.66 \mathrm{E}-005$
A) Nannoolal et al 2008

boiling point

$$
\begin{array}{lll}
a & b
\end{array}
$$

a

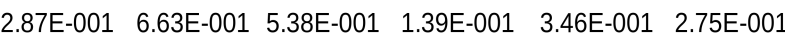

$\begin{array}{llllll}6.85 E-002 & 2.45 E-001 & 2.06 E-001 & 3.14 E-002 & 1.24 E-001 & 1.03 E-001\end{array}$

$\begin{array}{llllll}1.54 E-002 & 9.22 E-002 & 7.91 E-002 & 6.74 E-003 & 4.55 E-002 & 3.86 E-002\end{array}$

3.23E-003 3.54E-002 3.04E-002 1.38E-003 1.73E-002 $1.47 \mathrm{E}-002$

$\begin{array}{llllll}6.33 \mathrm{E}-004 & 1.39 \mathrm{E}-002 & 1.17 \mathrm{E}-002 & 2.70 \mathrm{E}-004 & 6.76 \mathrm{E}-003 & 5.61 \mathrm{E}-003\end{array}$

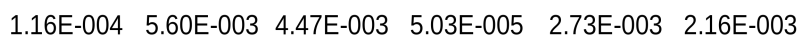

$\begin{array}{llllll}1.97 \mathrm{E}-005 & 2.26 \mathrm{E}-003 & 1.71 \mathrm{E}-003 & 8.94 \mathrm{E}-006 & 1.11 \mathrm{E}-003 & 8.35 \mathrm{E}-004\end{array}$

3.10E-006 8.86E-004 6.52E-004 1.52E-006 4.45E-004 3.25E-004

4.50E-007 3.39E-004 2.48E-004 2.46E-007 1.75E-004 1.27E-004

$\begin{array}{llllll}6.01 E-008 & 1.26 \mathrm{E}-004 & 9.38 \mathrm{E}-005 & 3.81 \mathrm{E}-008 & 6.71 \mathrm{E}-005 & 4.97 \mathrm{E}-005\end{array}$

7.37E-009 4.58E-005 3.53E-005 5.64E-009 2.53E-005 1.95E-005

$\begin{array}{llllll}8.25 \mathrm{E}-010 & 1.61 \mathrm{E}-005 & 1.32 \mathrm{E}-005 & 7.97 \mathrm{E}-010 & 9.36 \mathrm{E}-006 & 7.68 \mathrm{E}-006\end{array}$

8.40E-011 5.53E-006 $\quad 4.94 \mathrm{E}-006 \quad 1.08 \mathrm{E}-010 \quad 3.39 \mathrm{E}-006 \quad 3.03 \mathrm{E}-006$

$\begin{array}{llllll}7.74 \mathrm{E}-012 & 1.84 \mathrm{E}-006 & 1.83 \mathrm{E}-006 & 1.39 \mathrm{E}-011 & 1.20 \mathrm{E}-006 & 1.20 \mathrm{E}-006\end{array}$

$6.44 \mathrm{E}-013 \quad 5.96 \mathrm{E}-007 \quad 6.75 \mathrm{E}-007 \quad 1.72 \mathrm{E}-012 \quad 4.20 \mathrm{E}-007 \quad 4.74 \mathrm{E}-007$

$\begin{array}{llllll}4.81 \mathrm{E}-014 & 1.87 \mathrm{E}-007 \quad 2.47 \mathrm{E}-007 \quad 2.03 \mathrm{E}-013 & 1.43 \mathrm{E}-007 & 1.88 \mathrm{E}-007\end{array}$

$\begin{array}{llllll}3.20 \mathrm{E}-015 \quad 5.70 \mathrm{E}-008 & 9.01 \mathrm{E}-008 & 2.29 \mathrm{E}-014 & 4.80 \mathrm{E}-008 & 7.43 \mathrm{E}-008\end{array}$
Co) Compernolle et al 2011

for all boiling points

3.05E-001

9.98E-002

3.27E-002

1.07E-002

3.50E-003

1.15E-003

3.75E-004

1.23E-004

4.01E-005

$1.31 \mathrm{E}-005$

4.30E-006

$1.41 \mathrm{E}-006$

4.61E-007

1.51E-007

4.93E-008

$1.61 \mathrm{E}-008$

5.28E-009

595 Table 5-S. Vapour pressure data at $298 \mathrm{~K}$ from different sources corresponding to Figure 2 in the

597 Brown (1994) and Nannoolal et al. (2004), respectively. A-a, B-c and Co are used in this study. 
611 $\mathrm{C}_{16} \mathrm{H}_{34} \quad 6.42$ (3.62)

(6) $\mathrm{C}_{17} \mathrm{H}_{36}$ $9.32(4.31)$

612 $\mathrm{C}_{18} \mathrm{H}_{38}$ $9.36(4.41)$

613 $\mathrm{C}_{19} \mathrm{H}_{40}$ 9.74 (4.09) $\mathrm{C}_{20} \mathrm{H}_{42}$ 8.35 (3.59)

614 $\mathrm{C}_{21} \mathrm{H}_{44}$ $6.20(3.06)$

615 $\mathrm{C}_{22} \mathrm{H}_{46}$ 3.97 (1.98)

616 $\mathrm{C}_{23} \mathrm{H}_{48}$ $2.32(1.25)$ $\mathrm{C}_{24} \mathrm{H}_{50}$ 1.59 (1.79) $\mathrm{C}_{25} \mathrm{H}_{52}$ $1.20(0.52)$

617 $\mathrm{C}_{26} \mathrm{H}_{54}$ $1.00(0.50)$

618

619

$\mathrm{C}_{27} \mathrm{H}_{56}$ $1.28(0.53)$

$\mathrm{C}_{28} \mathrm{H}_{58}$ $1.03(0.53)$

620 $\mathrm{C}_{29} \mathrm{H}_{60}$ $1.24(0.53)$

621

$\mathrm{C}_{30} \mathrm{H}_{62}$ $0.80(0.38)$ $\mathrm{C}_{31} \mathrm{H}_{64}$ $0.77(0.30)$

622 $\mathrm{C}_{32} \mathrm{H}_{66}$ $0.42(0.18)$

623

624

625

Table 6-S. Gas-phase concentrations used in the model. Numbers in parenthesis show the 626 standard deviation of the measurements. Unit, $\mathrm{ng} \mathrm{m}^{-3}$.

627

628

629

630

631

632

633

634 